\title{
Supplemental Report: Application of Emission Spectroscopy to
} Monitoring Technetium

by

W. A. Spencer

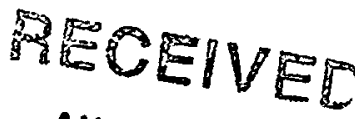

AUG 032080

Westinghouse Savannah River Company

Savannah River Site

Aiken, South Carolina 29808

This paper was prepared in connection with work done under the above contract number with the U. S. Department of Energy. By acceptance of this paper, the publisher and/or recipient acknowledges the U. S. Government's right to retain a nonexclusive, royalty-free license in and to any copyright covering this paper, along with the right to reproduce and to authorize others to reproduce all or part of the copyrighted paper. 


\section{DISCLAIMER}

This report was prepared as an account of work sponsored by an agency of the United States Government. Neither the United States Government nor any agency thereof, nor any of their employees, makes any warranty, express or implied, or assumes any legal liability or responsibility for the accuracy, completeness, or usefulness of any information, apparatus, product or process disclosed, or represents that its use would not infringe privately owned rights. Reference herein to any specific commercial product, process or service by trade name, trademark, manufacturer, or otherwise does not necessarily constitute or imply its endorsement, recommendation, or favoring by the United States Government or any agency thereof. The views and opinions of authors expressed herein do not necessarily state or reflect those of the United States Government or any agency thereof.

This report has been reproduced directly from the best available copy.

Available for sale to the public, in paper, from: U.S. Department of Commerce, National Technical Information Service, 5285 Port Royal Road, Springfield, VA 22161, phone: (800) 553-6847,

fax: (703) 605-6900

email: orders@ntis.fedworld.gov

online ordering: http://www.ntis.gov/ordering.htm

Available electronically at http://www.doe.gov/bridge

Available for a processing fee to U.S. Department of Energy and its contractors, in paper, from: U.S. Department of Energy, Office of Scientific and Technical Information, P.O. Box 62,

Oak Ridge, TN 37831-0062,

phone: (865)576-8401,

fax: (865)576-5728

email: reportseadonis.osti.gov 


\section{DISCLAIMER}

Portions of this document may be illegible in electronic image products. Images are produced from the best available original document. 
BNF-003-98-0233

Supplemental Report: Application of Emission Spectroscopy to Monitoring Technetium in Hanford Supernate Waste Solutions

TO: $\quad$ F. M. Pennebaker, 773-A

D. J. McCabe, $773-42 \mathrm{~A}$

R. A. Sigg, 773-41A

C. W. Jenkins, $773-41 A$

H. F. Strum, 773-A

FROM: $\quad$ W. A. Spencer, 773-41A (5-5256) 


\section{Supplemental Report: Application of Emission Spectroscopy to Monitoring Technetium in Hanford Supernate Waste Solutions.}

W.A. Spencer, F.M. Pennebaker, D.J. McCabe, R.A. Sigg, and C.W. Jenkins, Westinghouse Savannah River Company, Building 773A, Aiken, SC 29808

\section{DISCLAIMER NOTICE}

This report was prepared by Westinghouse Savannah River Company, Inc. (WSRC) on behalf of the U.S. Department of Energy (DOE), as an account of work sponsored by BNFL, Inc. Neither WSRC, DOE, the U.S. Government, or any person acting on their behalf makes any warranty, express or implied, or assumes any legal liability or responsibility for the accuracy, completeness, or usefulness of any information, apparatus, product, or process disclosed, or represents that its use would not infringe privately owned rights. Reference herein to any specific commercial product, process, or service by trade name, trademark, manufacturer, or otherwise, does not necessarily constitute or imply its endorsement, recommendation, or favoring by WSRC, DOE, or the U.S. Government. The views and opinions of authors expressed herein do not necessarily state or reflect those of WSRC, DOE, or the U.S. Government.

Savannah River Technology Center Westinghouse Savannah River Company Aiken, SC 29808 


\section{Approvals}
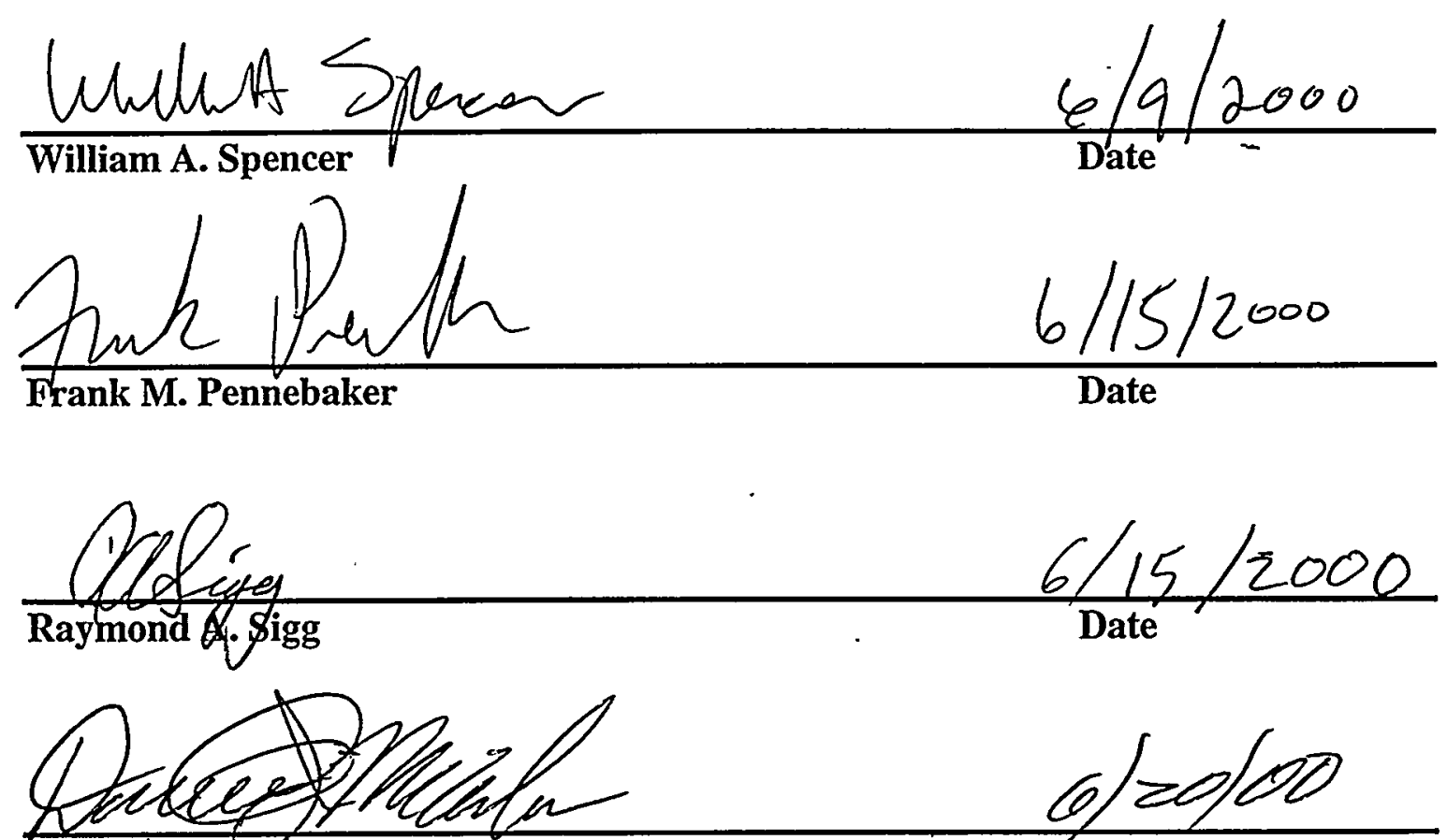

Daniel J. Mceabe, Technical Reviewer

Date

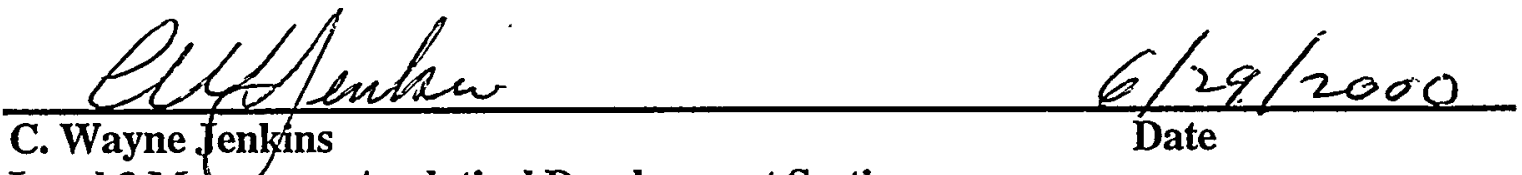

Level 3 Manager - Analytical Development Section 


\section{$\underline{\text { Summary }}$}

This report provides supplemental information to our earlier report, BNF-98-003-0199, "Evaluation of Emission Spectroscopy for the On-Line Analysis of Technetium". In this report we include data from real Hanford samples as well as for solutions spiked with technetium. This supplemental work confirms the ability of ICP-ES to monitor technetium as it breaks through an ion exchange process. Findings pertinent to accomplishing the monitoring mission are as follows:

- Measurement of real waste samples from Hanford validated previous work in that the sensitivity for technetium using the ICP-ES system is approximately an order of magnitude better for technetium than for rhenium. The detection limit for technetium varied between 0.05 and $0.12 \mathrm{ug} / \mathrm{mL}$ for real samples.

- A comparison of ICP-ES with ICP-MS and radiochemical methods showed similar results for Envelopes A, B, and eluted technetium in the water matrix.

Radiochemistry and ICP-ES report values that are $\sim 30 \%$ lower than those for ICPMS for the Envelope $\mathrm{C}$ matrix. The bias for the $\mathrm{C}$ samples did not affect breakthrough monitoring. The reasons for the bias in Envelope $C$ samples are still under investigation. Interference at mass 99 was investigated and not found.

- Design of hardware that will be in contact with radioactive materials was optimized. An all Teflon parallel flow nebulizer that was made by Burgener was preferred. Concentric nebulizers tended to plug. The double pass chamber appeared superior.

Additional studies concerning technetium monitoring were also made:

- A report on a laser spark excitation method that was investigated as a subtask by DIAL at Mississippi State is attached.

- The ICP-ES Tc monitor was used to observe effluents from SuperLig ${ }^{\circledR} 639$ column operations that mimic facility conditions. Elution with heated water showed that technetium was removed faster than with ambient temperature water. Optimum elution was accomplished with deionized water. Technetium appeared to be slightly more retained than rhenitm on the column.: 


\section{Introduction}

This report provides supplemental information to our earlier report, BNF-98-003-0199, "Evaluation of Emission Spectroscopy for the On-Line Analysis of Technetium" [refs. 13]. In this report we include data from real Hanford samples as well as for solutions spiked with technetium. The ICP-ES method was compared to ICP-MS and radiochemical methods. We include a report on the laser spark spectroscopy approach that was investigated as a subtask by Jagdish Singh and associates at the Diagnostic and Instrumental Analysis Laboratory (DIAL), located at Mississippi State University [ref 4].

While this work was on-going, we received a report on the use of NMR to measure Tc99 [ref. 5] issued by Dieckman, Jendrzejczyk, and Raptis at the Argonne National Laboratory. The report indicated that an approximately $2 \mathrm{ug} / \mathrm{mL}$ detection limit for pertechnetate ion was achievable using a 2T magnet. Sharma, Yueh, Singh, and Zhang at DIAL reported that a possible $1.0 \mathrm{ug} / \mathrm{mL}$ detection limit for technetium could be achieved using laser spark spectroscopy. DIAL analyzed the concentrations of manganese, rhenium, magnesium, and chromium in simulated Supernate solutions. They found that analysis from a side-stream supplied as a liquid jet stream worked better than analysis directly into a liquid solution.

The work reported in this paper was done in parallel with two other development efforts. First, SRTC continued to investigate direct flow-through beta scintillation methods. The beta counting approach primarily focused on combining simple chromatographic [ion exchange column] pre-processing of samples to separate interfering radionuclides from technetium. The second effort investigated an automated sampling station that quantitatively diluted samples and automatically added standards to provide an internallycalibrated sample which could be analyzed. The system inserted external standards into the flow path as well as did standard additions to process samples. Both of these efforts will be covered in separate technical reports [ref. 6].

\section{Experimental}

The technetium prototype analyzer has been previously described in our earlier report [ref 1]. The equipment is shown in Figures 1A-F. An Advanced Energy Products [formerly RFPP] ICP16L power supply and ICP transmatch provided the RF power for the plasma. Power was usually set at 1260 watts. A standard concentric torch with a $12 / 5$ male ball joint was used. Nominal argon flow rates were $15 \mathrm{~L} / \mathrm{min}$ to the outer sleeve, $0.5 \mathrm{~L} / \mathrm{min}$ to the inner sleeve cooling gas, and $1.2 \mathrm{~L} / \mathrm{min}$ through the nebulizer to the center feed tube. The gas feeds were controlled using Porter mass flow controllers. The plasma was monitored from the radial position $15 \mathrm{~mm}$ above the coil using a 1/2-inch quartz fiber optic lens assembly. The lens was mounted to the torch enclosure box and located approximately $10 \mathrm{~cm}$ from the torch. The lens connected to a 600 micron Polymicro quartz fiber that was connected to the entrance pinhole of a Thermo Jarrell Ash IRIS spectrometer. The fiber was 4 meters in length and allowed the spectrometer to be 
located outside of the radiological containment and buffer area. The torch and transmatch used a water recirculator to maintain cooling.

An Alltech model $426 \mathrm{HPLC}$ pump was used to pump distilled deionized water at 1.5 $\mathrm{mL} / \mathrm{min}$ through a Valco Cheminert, 6 port, 1/16-inch diameter fitting, sampling valve on to the nebulizer. The electronic controlled valve had an approximate $0.2 \mathrm{~mL}$ Teflon sampling loop that was filled with the effluent from a SuperLig ${ }^{\circledR} 639$ column or filled directly with test solutions. The valve connected the loop loading input to a waste collection or recycle point. For most of the work, a Burgener parallel flow standard nebulizer was used with a glass double-pass Scott type spray chamber.

The sampling valve control was tied to the TJA software for automatic synchronization with the emission analyses. The valve was switched at approximately 2-minute intervals and held in the injection state long enough to flush the injection loop before resetting to the loading position. Samples were usually pumped to the nebulizer at $1.5 \mathrm{~mL} / \mathrm{min}$ with water using the Alltech pump or a small gear pump. Delay and injection times-were modified as needed.

Analyses were started with $0.2 \mathrm{ml}$ injections of sample followed by an 8-second delay from the injection trigger. The delay allowed the loop contents time to reach the torch. The signal was then integrated for 10 seconds. With slower nebulizer pump flow rates the delay was lengthened to match the time to the torch. Detection of weaker deep UV lines could be improved with longer 30 and 60 second integration times but this required increasing the loop size to 0.5 and $1.0 \mathrm{~mL}$ or decreasing the flow rate respectively and increasing the time between analyses and the amount of flushing required. During column loadings, the delay time was easily determined by monitoring the bright orange color from sodium when the sample reached the torch.

Normal analysis with background corrected peaks could be programmed every 24 seconds. Elemental emissions were measured for 10 seconds and corrected for background emissions on each side of the emission. Data was automatically stored after each analysis. Approximately 30 elemental emission lines were routinely monitored simultaneously. Four emission lines were plotted in real time by the software. Normally a line for technetium $(253.4 \mathrm{~nm})$, sodium $(330.2 \mathrm{~nm})$, aluminum $(257.5$ or $308.2 \mathrm{~nm})$, and argon $(404.4 \mathrm{~nm})$ were monitored in real time.

Technetium was calibrated against an external standard purchased from NEN Life Science Products (\#NEZ085 - $17 \mathrm{mCi} / \mathrm{g}$ solid ammonium pertechnetate). The standard was dissolved in 2 percent nitric acid to make a $1000 \mathrm{ug} / \mathrm{mL}$ stock solution. This was scanned on ICP-MS for impurities $(<0.1 \%)$. Working standards were made from the stock solution and diluted to make a $10 \mathrm{ug} / \mathrm{mL}$ working solution. Working solutions were checked for expected activity using beta scintillation against NIST traceable beta standards. The Tc standards agreed with ICP-MS values that were based on molybdenum response as described below. 
For nebulizer efficiency studies raw real time elemental emission signals were tracked directly at approximately 0.1 second intervals using the TJA time scan function. The time scan mode enabled viewing of the sampling elution profile under different pump flow rates and integration times. Because of its fast response the time scan mode was also useful for aligning the fiber on the torch. A strong aluminum or sodium emission was usually monitored during these operations. The time scan mode was not useful for routine analyses because it did not background correct the signal and was somewhat affected by the integration reset of high count rate emissions. Figure $2 \mathrm{~A}$ shows the time scan Al profile for a $0.2 \mathrm{~mL}$ sample for both a Burgener and Hildebrand Grid nebulizer. Figure 2B shows the effect of the flow rate on the sample reaching the nebulizer.

For column elution studies, the BNFL designated waste simulants, types A, B, and C, were spiked with technetium-99 and rhenium. The Tc concentration was adjusted to between 2 and $15 \mathrm{ug} / \mathrm{mL}$ and rhenium was adjusted to between 10 and $30 \mathrm{ug} / \mathrm{mL}$. The higher rhenium was needed to increase the signal above noise levels for reasonable breakthrough times. The spiked simulants were pumped onto a $5 \mathrm{ml}$ Bio-Rad MT-5 column loaded with SuperLig ${ }^{\circledR} 639$ resin. The column was usually conditioned with at least 3 bed volumes of $1 \mathrm{M} \mathrm{NaOH}$ before and after loading simulant. Water was used to elute the technetium. The Tc was eluted until the starting baseline level was approached before recycling with another conditioning step. Effluent from the column passed through the sampling loop. Loading and conditioning solutions were pumped at $1.2 \mathrm{~mL} / \mathrm{min}$ - (approximately 15 column volumes per hour). These small column runs agreed well with runs on larger SRTC laboratory and pilot scale systems.

\section{Laboratory Analysis Method Description for SRTC Tc Analyses}

\section{Technetium (Tc) Analysis by Inductively Coupled Mass Spectrometry (ICP-MS)}

All measurements are performed on a VG Elemental Plasma Quad 2 quadrupole ICP-MS. The instrument is operated in scanning mode from mass 81 to mass 249 with a dwell time of $320 \mu \mathrm{s}, 20$ channels/AMU, and the detector in pulse counting only. The ICP-MS is calibrated with a blank and three multi-element standards, containing Molybdenum (Mo), of concentrations: 1,10 , and $100 \mu \mathrm{g} / \mathrm{L}$. All samples and standards contain $50 \mu \mathrm{g} / \mathrm{L}$ Indium (In). An internal standard correction is performed on all samples and standards using mass 115 of In. A blank subtraction is also applied to all standards and samples. Sensitivity for $\mathrm{Tc}$ is derived from Mo isotopes in the multi-element standards. The derived Tc sensitivity is used to calculate the concentration of Tc in the samples analyzed. Final results are compensated for any dilutions performed prior to analysis.

\section{Technetium (Tc) Analysis by $\beta$-Counting}

One mL samples were spiked initially with $\sim 1000 \mathrm{~Bq}$ of $\mathrm{Tc}-99 \mathrm{~m}$ to act as a tracer for the separation process. The samples were then acidified to $\mathrm{pH}<2$ with $2 \mathrm{~N}$ nitric acid, and the Cs- 137 levels in the samples were lowered by adding about 0.1 gram of ammonium molybdo-phosphate solid (Bio-Rad AMP), swirling, and filtering. The Tc-99 in the 
samples and the Tc-99m in the tracer were subsequently reduced with $5 \mathrm{M}$ hydroxylamine hydrochloride that was catalyzed by addition of $0.1 \mathrm{~mL}$ of $0.025 \mathrm{M}$ ferric nitrate. The samples were then oxidized using 30 percent hydrogen peroxide catalyzed with sodium vanadate [ $2 \mathrm{~mL}$ of a $20 \mathrm{~g} / \mathrm{L}$ solution] at 80 degrees $C$ for 1 hour. Oxidized solutions were diluted and the Tc was extracted from the solutions with a solvent/solvent extraction based on 30 percent Aliquat-336 in xylene. The organic phase was washed sequentially with $10 \mathrm{~mL}$ of a $1 \mathrm{M}$ nitric acid solution, followed by a $4 \mathrm{M} \mathrm{NaOH}$ caustic wash. An aliquot of the organic phase was added to liquid scintillation cocktail (Ultima Gold AB [Packard]) and analyzed for Tc-99 by liquid scintillation, and by gamma spectroscopy to determine the Tc-99m recoveries. The gamma analysis results were also used to ensure no other gamma-emitting radionuclides were present in the Tc-99 organic phase. A blank was run through the process for each batch of samples analyzed. The Tc$99 \mathrm{~m}$ recoveries were used to correct the liquid scintillation analyses results for the Tc-99 recovéries.

\section{Technetium (Tc) Analysis by ICP-ES .}

Two ICP-ES instruments were used to collect data on technetium for these BNFL/Hanford samples. The first was a contained ARL 3580 instrument, which included both a sequential and simultaneous spectrometer. This system is used for bulk sample analysis in our Analytical Development Section. The other was a hybrid system that combined a commercial TJA IRIS echelle spectrometer with a remote ICP source via fiber optic cable described earlier

Hanford samples were diluted 5X for envelope "large C" and 20X for envelope B and spiked to a final concentration of $2 \mathrm{mg} / \mathrm{L}$ with Sc. The Tc was analyzed at $264.7 \mathrm{~nm}$ and $254.3 \mathrm{~nm}$ in both instruments and at $261.0 \mathrm{~nm}$ for the TJA system. The Tc standards were prepared from a stock solution of $1000 \mu \mathrm{g} / \mathrm{mL} \mathrm{Tc}$ diluted and calibrated against a NIST standard by $\beta$-counting. Scandium was used for internal standard correction at Sc $361.4 \mathrm{~nm}$ for both instruments. Alternate Sc wavelengths at $357.2 \mathrm{~nm}$ and $363.0 \mathrm{~nm}$ were measured to verify the internal standard correction. Results in the graph were reported from the ARL 3580 using Tc $264.7 \mathrm{~nm}$ due to known interference from Cr at $254.3 \mathrm{~nm}$.

The TJA ICP-ES used two sided background correction for the $254.3 \mathrm{~nm}$ and $261.0 \mathrm{~nm}$ emission lines and single sided correction for the $264.7 \mathrm{~nm}$ line. The latter was necessary to decrease the effect of molybdenum line interference at $264.65 \mathrm{~nm}$. The ARL system did not correct for background emission or interfering line emissions adjacent to the peak of interest.

We attempted to correct for line interference using the TJA sub array software, which subtracted average readings on each side of the monitored pixels from the background emission. In the TJA system, a 15 pixel wide by 3 pixel high sub array was centered on each monitored emission line. The peak was measured from the integrated intensity at the center $3 \times 3$ pixels. Intensity from the $1 \times 3$ pixels on the wings of the mask was used to correct for the baseline emissions. The technetium $264.70 \mathrm{~nm}$ line had a slight interference on one side from molybdenum at $264.65 \mathrm{~nm}$ and the background on that side 
was not used. This line had a slight negative bias. The $254.3 \mathrm{~nm}$ line had a small positive bias compared to the other samples and was likely interfered with by a general background shift that occurred due to the intense aluminum line emissions in the orders above at 256.798 and $257.510 \mathrm{~nm}$ and an interference with chromium. The Al lines would likely not interfere on a different echelle grating type or if a conventional dispersive spectrometer were used. The $261.0 \mathrm{~nm}$ line appeared to work well with the large $C$ envelope sample but normally would have interferences from iron and. manganese, and possibly from lanthanum and rhenium.

\section{Results and Discussion}

\section{Technetium Emission Spectrum and interferences}

Table 1 compares the count rate for manganese, rhenium, and technetium for a $10 \mathrm{ug} / \mathrm{mL}$ solution of each element. As expected the technetium lines were more sensitive than rhenium emissions and less sensitive than the best manganese line. In the ICP, the technetium triplet ion lines at 254.3,261.0, and $264.7 \mathrm{~nm}$ dominated the Tc emission spectrum. A weaker triplet was easily observed at $319.5,321.2$, and $323.7 \mathrm{~nm}$. Neutral lines at $429.7,426.65$, and $423.8 \mathrm{~nm}$ were observed in the spectrum but weak and noisy due to the large plasma background. Figures $3 \mathrm{~A}$ and $3 \mathrm{~B}$ show the full technetium spectrum in the TJA echelle after the plasma background has been subtracted. Figure 3B is a slightly enlarged image to enhance the technetium lines. Figure $3 \mathrm{C}$ shows the spectrum for the neutral lines and the higher wavelength ion lines from $300-450 \mathrm{~nm}$. Figure 3D shows the more intense UV ion emission lines from $245-330 \mathrm{~nm}$.

Changes to the power level from 700 to 1350 watts did not shift the line intensities between neutral and ion lines at the normal monitoring location. We had some evidence that in very high salt samples that the neutral line intensities increased relative to the ion lines but because of the large sodium emissions this was not fully studied. Like manganese neutral lines, technetium neutral lines were enhanced in higher and outer regions of the torch plasma.

One interesting technetium line occurred at $249.68 \mathrm{~nm}$. This line on our spectrometer fell on top of the boron doublet line at $249.67 \mathrm{~nm}$ and would interfere with boron analyses if that line were used for the analyses. The $249.68 \mathrm{~nm}$ Tc line had about 10 percent of the intensity of the $254.3 \mathrm{~nm}$ line. 
Table I -- Relative Intensity on an Echelle Spectrograph of Mn, Tc, and Re ion lines.

\begin{tabular}{|lr|}
\hline $10 \mathrm{ug} / \mathrm{mL}$ & Count rate \\
& \\
Mn2576 & 35.76 \\
Mn2593 & 160.30 \\
& \\
Re2214 & 2.88 \\
Re2275 & 3.20 \\
Re3451 & 1.01 \\
Re3464 & 1.62 \\
& \\
Tc2543 & 36.49 \\
Tc2610 & 25.89 \\
Tc2647 & 24.76 \\
Tc3237 & 12.46 \\
\hline
\end{tabular}

Figures 6 A, B, and C show technetium measurements during column loading and elution operations with a simulated Envelope A material. The figures are from the same run and show different ways of looking at the data using the ICP-ES instrumentation. Figure 6B shows the effects of background shifts on key lines during the column loading operations. Envelope A simulant is based on Hanford AN-105 waste tank and is very high in aluminum and sodium. The $261.0 \mathrm{~nm}$ line appeared to have the least line bias from the high salt samples. The TJA IRIS used two-sided correction for the $254.3 \mathrm{~nm}$ and $261.0 \mathrm{~nm}$ emission lines and single sided correction for the $264.7 \mathrm{~nm}$ line. The latter was necessary to decrease the effect of molybdenum line interference at $264.65 \mathrm{~nm}$. The ARL system, used for lab measurements, did not correct for background emission or interfering line emissions adjacent to the peak of interest.

Detection limits for technetium in simulated wastes were determined between 0.05 and $0.12 \mathrm{ug} / \mathrm{mL}$ and were dependent on the pump type, plasma settings, and which nebulizer/chamber was being used. Detection in pure water or nitric solutions was as low at $0.02 \mathrm{ug} / \mathrm{mL}$. MDL's could be improved by using longer integration times or by averaging data with a subsequent loss in time resolution. Although not readily shown, technetium was easier to detect with more sensitivity in the elution operation than during the loading cycles. Scandium was found to be a good internal standard to adjust for the sensitivity shifts and was used for lab measurements. However, we judged that it was not needed for column monitor runs since relative-count rates can give a good indication of breakthrough. All measurements were made using radial [side view] emission detection. Because of the very high salt content of the load solution, axial viewing was not tried although it might improve the detection limits.

We found that some of the noise in the measurements was due the HPLC pump. Noise levels were decreased about 50 percent by switching to a gear pump instead of an HPLC pump to feed the nebulizer. Unfortunately, the gear pump did not maintain a constant flow rate under all loading conditions and did not have over pressurization safety shut 
offs and back pressure indicators. The back pressure reading was useful for detecting plugged nebulizers as mentioned below.

\section{Technetium versus Rhenium}

Figures $7 \mathrm{~A}$ and $\mathrm{B}$ show the same data at two different scale factors for another column run. Rhenium appeared to breakthrough the column at a higher molar rate tharr technetium although concentrations differed. In this run technetium in the feed was 10 $\mathrm{ug} / \mathrm{mL}$ while rhenium was spiked at approximately $25 \mathrm{ug} / \mathrm{mL}$, to compensate for low wavelength light losses in the fiber. Only the initial stages of breakthrough are shown in the two-hour load cycle. The solution was pumped at $1.2 \mathrm{~mL} / \mathrm{min}$ and the column volume was $5 \mathrm{~mL}$. About 24 column volumes of solution were pumped through the resin, and the calculated saturation point for $\operatorname{Re}$ and Tc was about 200 column volumes. A small amount of both elements passed through the column in this short experiment, due to the high flow rates.

\section{System Parameters}

\section{Nebulizers}

Several different nebulizers were evaluated for use with the caustic sample envelopes. An all Teflon ${ }^{\circledR}$ Burgener ${ }^{\circledR}$ parallel flow nebulizer was preferred. The Burgener gave about $80 \%$ of the response compared to a Hildebrand Grid [Leeman] nebulizer but had only half the noise for an overall signal to noise increase. This is illustrated for the Al $308.2 \mathrm{~nm}$ wavelength in Figure 2A. Both the Burgener ${ }^{\circledR}$ and the Hildebrand Grid [Leeman] handled high solids well and did not plug during months of operation. Both were more robust than a conventional cross-flow nebulizer.

Attempts to use concentric nebulizers failed. We evaluated both a CPI PFA-ST inert Teflon $^{\circledR}$ concentric nebulizer as well as a Glass Expansion PolyCon concentric nebulizer. Both nebulizers failed because solids precipitated from the solutions under some of the operating conditions and plugged the concentric nebulizers. In undiluted simulant; aluminum would precipitate if contacted with the water flush or acid rinse solutions. Due to the critical dependence on maintaining a high $\mathrm{pH}$ to avoid precipitiation, a robust nebulizer such as the Burgener ${ }^{\circledR}$ is recommended for Tc monitoring.

Figure 2B demonstrates the sample elution profile through the nebulizer/spray chamber for $\mathrm{Al}$ emission in real time. The Burgener nebulizer exhibited less tailing than the Hildebrand. When properly adjusted to flow rate, the rectangle input plug flow became a slightly distorted rectangle output function that had a small elution tail. Slowing the flow rate increased the curvature on the front and back ends and decreased the overall maximum intensity of the signal as the signal size widen. The total area under the signal remained nearly constant but the instantaneous signal to noise ratio decreased with slower flow rates. 


\section{Spray Chambers}

Both a glass double pass [tube within a tube], and a Teflon ${ }^{\circledR}$ cyclonic nebulizer were evaluated. The double pass appeared to work a little better. The cyclonic provided less signal and lower noise. Neither had a significant advantage. The double pass appeared to tail less and have shorter resonance time. That is, it had a better response to transient signals. That effect might have been due to incorrect flow settings to optimize-the cyclonic nebulizer as they were set by simple trial and error adjustments. The cyclonic might work better if we had enough sample to fully load the chamber and allow resonance to set up. The glass double pass spray chamber did not appear to be significantly affected by the caustic samples. Silicon background was not high in the blanks and the chamber did not appear to be etched after months of use. Either spray chamber design would be appropriate for an on-line system.

\section{Column Studies}

\section{Temperature Run}

The system was used to observe several SuperLig ${ }^{\circledR} 639$ column performance parameters. Figures $4 \mathrm{~A}$ and $\mathrm{B}$ show elution with heated water. As expected, rhenium was removed faster with $60^{\circ} \mathrm{C}$ water.

\section{Elution Studies}

As shown in Figures 5A and 5B, several different elution conditions were tried. In each of these runs a $5 \mathrm{~mL}$ SuperLig ${ }^{\circledR} 639$ column was conditioned with $1 \mathrm{M} \mathrm{NaOH}$, then loaded with an Envelope A simulant that contained rhenium. Solutions were pumped at $1.2 \mathrm{~mL} / \mathrm{min}$. After loading, the simulant solution was flushed from the column with $1 \mathrm{M}$ $\mathrm{NaOH}$ prior to elution of the technetium using a test elution solvent. Water was used after the elution solvent to remove any retained technetium. As shown in the figures the sodium emission response could be used to indicate the elution stage. Elution with $0.5 \mathrm{M}$ acid showed a spike for several transition metals and strontium. It was believed that the higher level of acid elution dissolved micro particulates that had collected on the column. With high acid concentration, technetium tended to elute with a long tail. Technetium had a significant tail with $0.05 \mathrm{M}$ acid.

The overall technetium level in the $0.05 \mathrm{M}$ acid elution run was lower than expected. We speculate that the feed solution to the SuperLig ${ }^{\circledR}$ column might have had a partial plug and less technetium was loaded onto the column. Flow to the ICP-ES nebulizer was tightly controlled and monitored by back pressure and displacement using a HPLC pump but flow to the SuperLig ${ }^{\circledast}$ column was not monitored and subject to some fluctuations due to the experimental set up. A decreased column flow rate was not readily detected using the ICP-ES, as concentration going to the sampling loop would change slowly. After several experiments we learned that a low flow condition could occur during column loading but when the water and caustic flush were used the flow rate would often 
recover. A flow rate sensor on the column system would have detected this problem. When flow completely stopped in a way that the sampling loop was not filled, the ICPES did detect a loss of signal. This situation is shown in the last water elution run. This problem is not expected to be present in the Hanford facility, as the system will be controlled and instrumented. It does indicate one type of problem that can occur if a sampling line is plugged and the sample in the lines does not indicate the actual column effluents.

Of the solutions tested, optimum elution occurred with deionized water. In deionized water technetium eluted with the least tailing peak and more was released from the column. Using $0.05 \mathrm{M} \mathrm{NaOH}$ for the technetium column flush caused some of the technetium to elute but some remained bound to the column. After the dilute caustic, a second technetium peak was observed when the column was subsequently flushed with deionized water.

An interesting side phenomenon is shown in Figure 5B. Trace strontium appears to be collected from the $1 \mathrm{M} \mathrm{NaOH}$ conditioning solutions and then eluted in the stronger 2 to $5 \mathrm{M} \mathrm{Na}$ envelope solutions. A second observation is that some potassium appears to be retained in the initial contact of the SuperLig ${ }^{\circledR}$ column with the envelope A solution. Potassium appears to reach an equilibrium concentration more slowly than other metals like aluminum and sodium. We are not sure though if these changes are due to the effects of the high salt matrix on the plasma, i.e. instrumental problem, or real changes on the SuperLig ${ }^{\circledR}$ column.

\section{Analysis of Hanford Waste and Comparison with Other Systems}

In our earlier report (reference 1) and earlier in this document, all $\mathrm{Tc}$ and $\mathrm{Re}$ analyses were performed on simulants spiked with Tc and Re. In this section, we discuss the ability to monitor Tc in actual Hanford samples. Solutions were readily available from SRTC Tc decontamination experiments for envelopes B and "Large" C. Solutions were also available undiluted from the SRTC Shielded Cells because of the earlier Cs-137 decontamination and Sr-TRU removal. This section will focus mainly on envelope "large C," tank AN102, because decontaminated product from envelope B was below detection limits for ICP-ES. The ICP-MS measured Tc at $7 \mathrm{ng} / \mathrm{ml}(\mathrm{ppb})$ in the decontaminated product. Technetium eluted from the column for envelope B was measured by both ICPES and ICP-MS. Results agreed within $9 \%$.

Figure 8 shows a comparison of measurements made on the prototype ICP-ES Tc monitor with samples analyzed on the standard lab ARL 3580 ICP-ES system. These samples were fractions taken from a "Large" $C$ column run that used Hanford Tank AN102 as the loading solution. As shown, the analyses between the two different ICP-ES systems agreed fairly well. The average deviation was $4 \%$.

Figure 9 compares Tc measurement by ICP-MS with those of the lab ICP-ES and radiochemistry. Figure 10 compares Tc measurements for some ICP-MS and 
radiochemical analyses samples. The latter were more noisy and appeared to be plagued by occasional radioactive contaminants.

Three of the samples from "Large" C Tc-99 studies were fully oxidized before radiochemical extractions. The results were compared to the ICP-MS and ICP-ES results. For feed material, the $\beta$-counting Tc measurements were in between the ICP-ES and ICP-MS results. For two product samples [water elution matrix], ICP-ES-and ICPMS were very close. The ICP-MS tended to report a higher value for Tc than $\beta$-counting. In the ICP-ES and ICP-MS comparison above, we found that ICP-MS produces higher values than measurements using an ICP-ES method. Furthermore, we have found that the difference is only significant in the high organic envelope " $C$ " fractions.

Envelope "Large" C, AN-102, is somewhat different from the other Envelope C tank (AN-107) in that it has a higher aluminum concentration and lower concentrations of transition metals [Fe, $\mathrm{Ni}, \mathrm{Mn}$ ]. The sample is high in soluble organic levels and has about two thirds of the Tc in a non-pertechnetate form. The envelope has a high concentration of $\mathrm{Sr}$ due to an isotopic dilution with natural strontium during a prior treatment to remove Sr-TRU via precipitation. While treating the "Large" C envelope, the SuperLig $^{\circledR}$ column removed only a third of the technetium. This means that Tc will not be completely removed by the SuperLig ${ }^{\circledR}$ column and that technetium was detectable in the fractions breaking through the column.

The graph in Figure 11 compares ICP-ES and ICP-MS. Tc measurements from fractions taken during loading of a SuperLig ${ }^{\circledR} 639$ column. The high concentration of organics, $\mathrm{Al}$ and $\mathrm{Na}$ combined for severe matrix effects on the ICP-MS data. Note the sample-tosample fluctuation for samples 7-14. While there was a bias of $20-30 \%$ between methods, ICP-ES and ICP-MS showed similar trends in the data. As an example during measurement of the first two samples, both ICP-MS and ICP-ES were measured low as expected due to column mixing with the $\mathrm{NaOH}$ pretreatment. The concentrations went up in both methods when undiluted material was collected.

SRTC looked for interferences at mass 99 in the ICP-MS method but did not find any significant ones. An early thought to explain the higher results of the ICP-MS method was that a natural abundance ruthenium peak occurred at mass 99 , but data do not support that this is the source of the discrepancy. Figure 12 shows results for a simulant of envelope $C$, from masses 94-100. The simulant was run without technetium, i.e. a mass at 99 would be due to interference. As expectedd mass 99 was not observed indicating that a 99 peak due to molecular combinations of organics or lighter elements was not formed. A second simulant was spiked with Sr to compensate for the effects of the isotope dilution with natural Sr. There was no interfering peak observed at mass 99 for this sample either. Please note that the simulant was derived from tank AN-107, a different envelope $C$ tank than that used for the "Large C" studies.

The data shown in Figure 13 from SuperLig ${ }^{\circledR}$ column runs using Envelope A and B solutions showed that the mass 99 peak was efficiently removed. That is, the residual 99 peak in the effluent of those runs was much less than the $30 \%$ bias seen in the Large $C$ 
runs. Envelopes A and B have technetium mostly in the pertechnetate form and the column removes that form of technetium as indicated by the small mass 99 . Figure 13 shows that any interference is at most $3 \%$ of the original mass 99 signal in envelopes $\mathrm{A}$ and $B$ feed material. The residual mass- 99 signal in the fractions is believed to be Tc in the non-pertechnetate form or small amounts of technetium pertechnetate that is breaking through the column. In contrast for the "Large" $\mathrm{C}$ runs the mass 99 peak in the fractions was about two thirds of the original mass 99 peak indicating that only 30-40\%.of the Tc99 is removed for envelope $C$. This indicates that any interference at mass 99 is unique to the $\mathrm{C}$ type of envelopes.

Figures 14-16 show efforts to find possible mass interferences that might explain the higher ICP-MS results. We did not find them. The ICP-MS spectrum from mass 94-104, including Tc-99, for envelope large $\mathrm{C}(\mathrm{AN}-102)$ is shown in Figure 14. This sample has been decontaminated for $\mathrm{Cs}$ and $\mathrm{Sr}$ and TRU elements.

ICP-MS measured masses 95-104 in 10 samples during Tc decontamination. The results are tabulated in concentration (Figure 15) and Ru and Mo isotopic distributions (Figure 16). While $\mathrm{Mo}$ and $\mathrm{Ru}$ had other natural isotopic abundances, we only used isotopes specific for fission products. The isotope ratios for $\mathrm{Mo}$ and $\mathrm{Ru}$ matched expected fission product isotope ratios within 5\% with the exception of mass 104. An interference of SrO is present at mass 104 because the processed tank contained a high concentration of $\mathrm{Sr}$ $(\sim 100 \mathrm{ug} / \mathrm{mL})$. Interference from $\mathrm{Zn}, \mathrm{Cl}, \mathrm{Sr}$ and other elements with distributions of isotopes was not indicated.

Bias in the ICP-ES method is still somewhat unknown due to the limited testing that we have done. One possible explanation for the bias in the methods is due to the oxidation state. The technetium that is being measured in the problem C envelopes is mostly in the non-pertechnetate form. The SuperLig ${ }^{\circledR} 639$ column removed the pertechnetate form from these samples. Technetium in the samples required oxidation for radiochemical analyses methods.

In the ICP-ES measurements, we assumed that the plasma temperature would quickly destroy any Tc complexes and that the oxidation state of technetium did not matter. This might be an invalid assumption. The technetium standard, which was used to calibrate the ICP-ES, was in the pertechnetate form, which is the only form commercially available. The non-pertechnetate forms are likely part of an organic complex and might take longer to decompose in the plasma therefore decreasing the rate of atomization. One proposed test is to synthesize a non-pertechnetate standard and compare it to the pertechnetate one. Increasing the power to the plasma and adding some air to the argon to increase oxidation of the organic load might also decrease the bias. We had some indication that in Envelope $\mathrm{C}$ runs the plasma became less ionizing. However measurements of the neutral lines was hindered by the large change in the background signal that occurred in the plasma due to the sodium matrix and low dilution levels.

On a limited basis, ICP-ES was used to analyze envelope B material. In direct comparison of the product and eluate from the resin, ICP-ES and ICP-MS are consistent 
within $10 \%$. Column eluate consisted primarily of Tc-99 with a greatly reduced sodium and $\mathrm{Al}$ concentration. The feed material for the Tc resin would provide a better comparison in the future.

To summarize, a concern for BNFL has been the possibility of interference at mass 99 , giving a biased high value for Tc-99. SRTC looked for interferences at mass 99 in the Hanford waste tanks samples and found little or no interference in the tank samples at mass 99. Radiochemical and ICP-ES methods still indicate a 20-30\% lower amount for technetium in the Envelope $\mathrm{C}$ samples compared to measurements by ICP-MS. A smaller difference between methods was observed for Envelopes A and B samples. Obviously, more data is needed to definitively prove a low bias in the two methods compared to ICP-MS. Our studies to date have mainly focused on being able to track relative changes in the technetium concentrations with the ICP-ES monitor rather than on the accuracy of discrete measurements. The ICP-ES has been successful at tracking the changes. That is, we wanted to monitor column breakthrough and were not trying to determine exact Tc numbers. Bias corrections will need to be developed as experience is obtained. 


\section{References}

1. W.A. Spencer, F. M. Pennebaker, N.M. Hassan, J.E. McCarty, and C.W. Jenkins, "Evaluation of Emission Spectroscopy for the On-Line Analysis of Technetium", BNF-003-98-0199 Rev 1, Feb.7, 2000.

2. W.A. Spencer, J.C. Griffin, R.A. Dewberry, J.C. Hart, L.L. Tovo, C.J. Coleman, R.J. Ray, F.M. Pennebaker, J.R. Elliott, R.J. Lascola, J.D. Leyba, R.A. Sigg, J. Olson, T.L. White, A.A. Ekechukwu, "BNFL Interim Report on Candidate Technetium Analyzers", BNF-003-98-0041 Rev 1, Feb. 22, 1999.

3. Technical Task Plan and QA Plan, "On-Line Technetium Monitor: A Subtask for Savannah River Technology Center to Support Processing of Hanford Waste by BNFL", BNF-003-98-0020, Nov. 1998.

4. Ramesh C. Sharma, Fany-Yu Yueh, Jagdish P. Singh, and Hansheng Zhang, "Quantitative Measurement of Chromium and Magnesium in Liquid by Laser-induced Breakdown Spectroscopy", Grant No. Task GA0046, Final Report, Diagnostic Instrumentation and Analysis Laboratory, Mississippi State University, May 29, 2000.

5. S.L. Dieckman, J.A. Jendrzejczyk, and A.C. Raptis, "Development of a Magnetic Resonance Sensor for On-Line Monitoring of 99Tc and $23 \mathrm{Na}$ in Tank Waste Cleanup Processes: Final Report and Implementation Plan", ANL-ET/99-17, Argonne National Laboratory, Argonne, Mlinois 60439.

6. Contacts are Raymond Sigg for beta measurements and Michael Whitaker for sampling systems, Westinghouse Savannah River Company, SRTC, Aiken, SC.

7. W. F. Meggers, "Multiplets and Terms in Technetium Spectra", J. Res. Nat. Bur. Stds., v47, 7-14, 1951.

8. "Tc-99 Analysis in HLW Samples", Telephone Conference Call [Workshop], May 3, 2000. Contact: Karyn Wiemers, Pacific Northwest Laboratories or Louis Kovach, BOE BAT/ORP, Richland, Washington for meeting notes. 


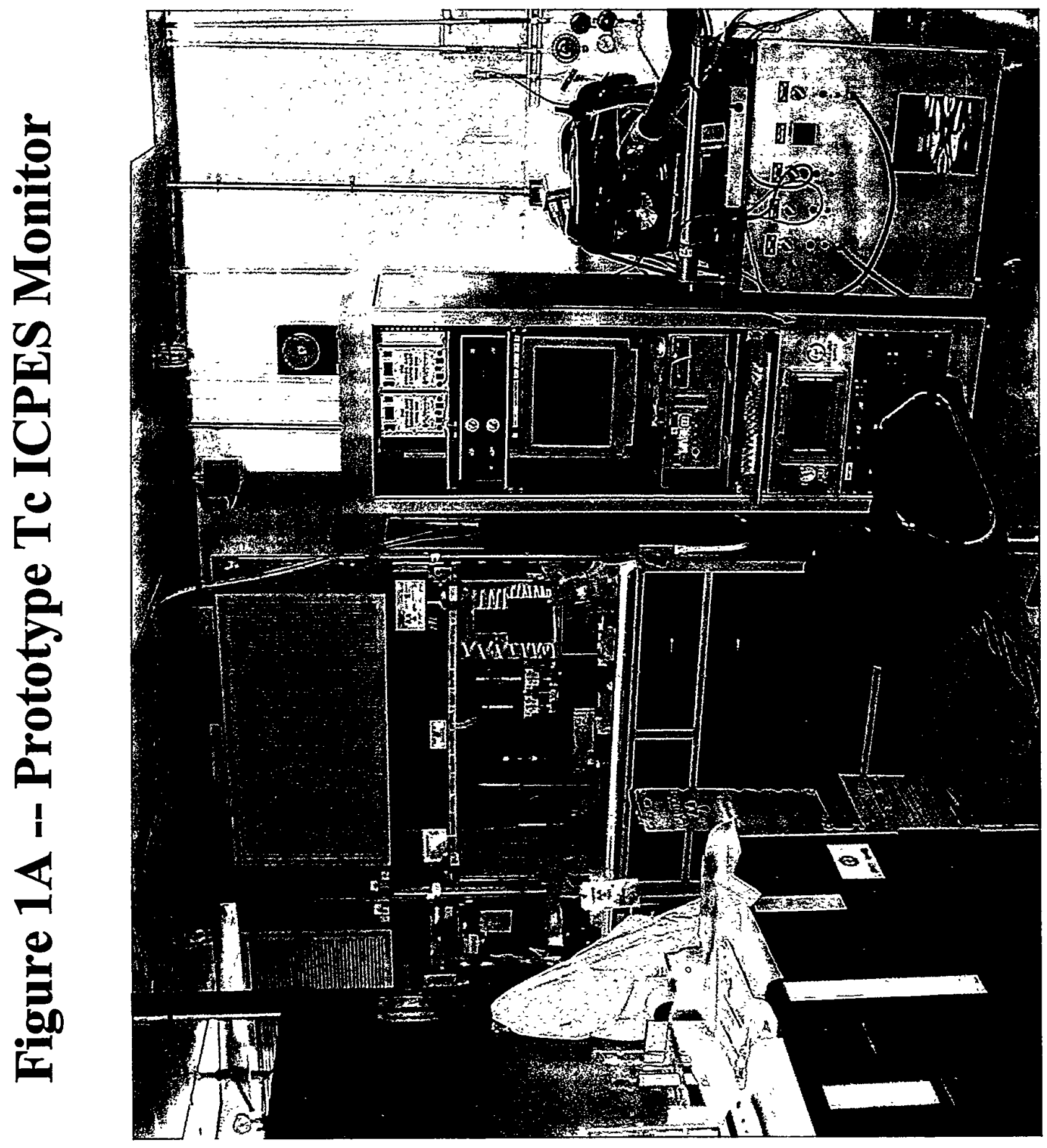


BNF-003/-0233

REV. 0

PAGE 18

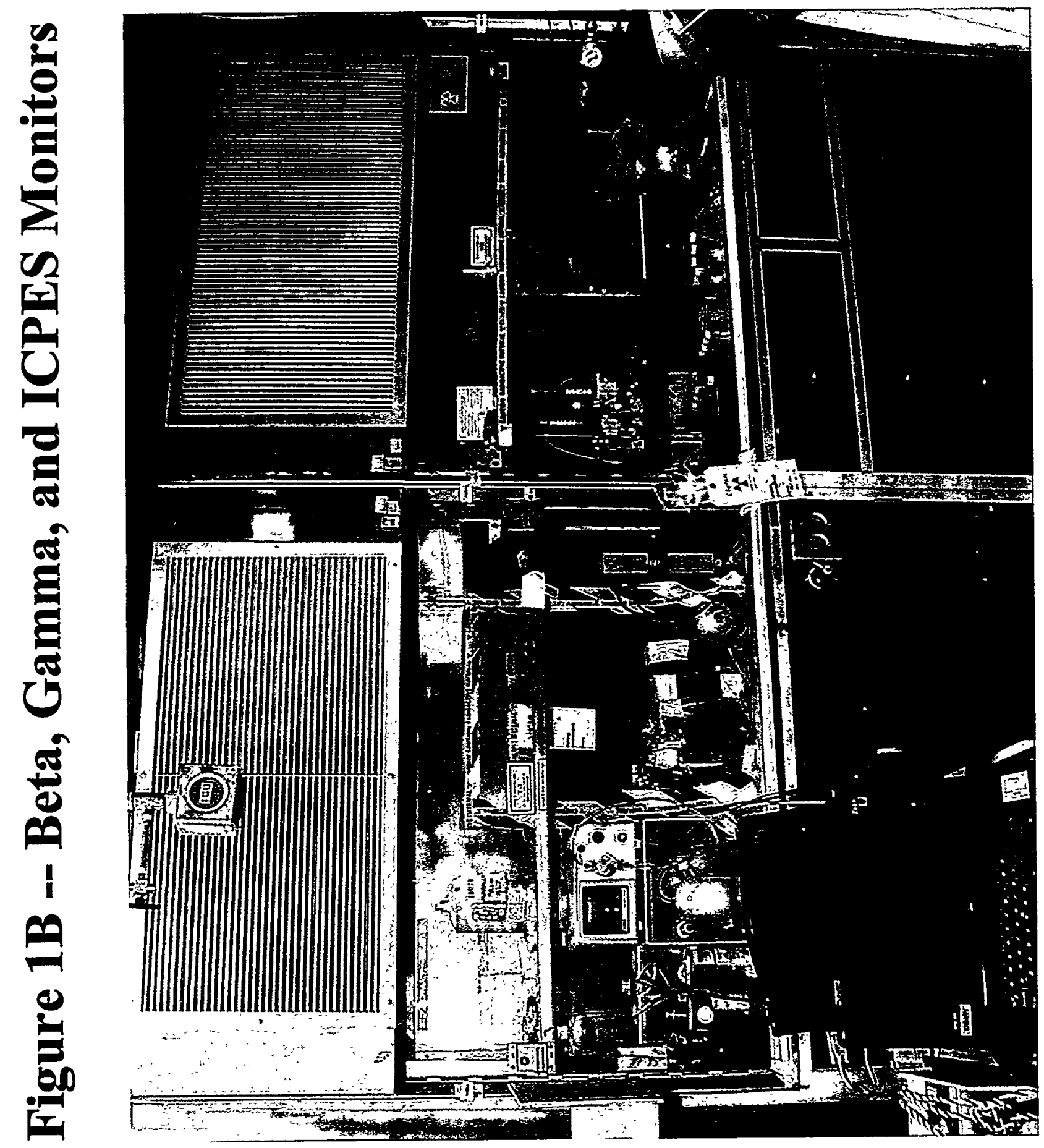




\section{Figure 1C}

\section{Alltech HPLC Pump, Packard Beta Monitor, NaI Well Detector}

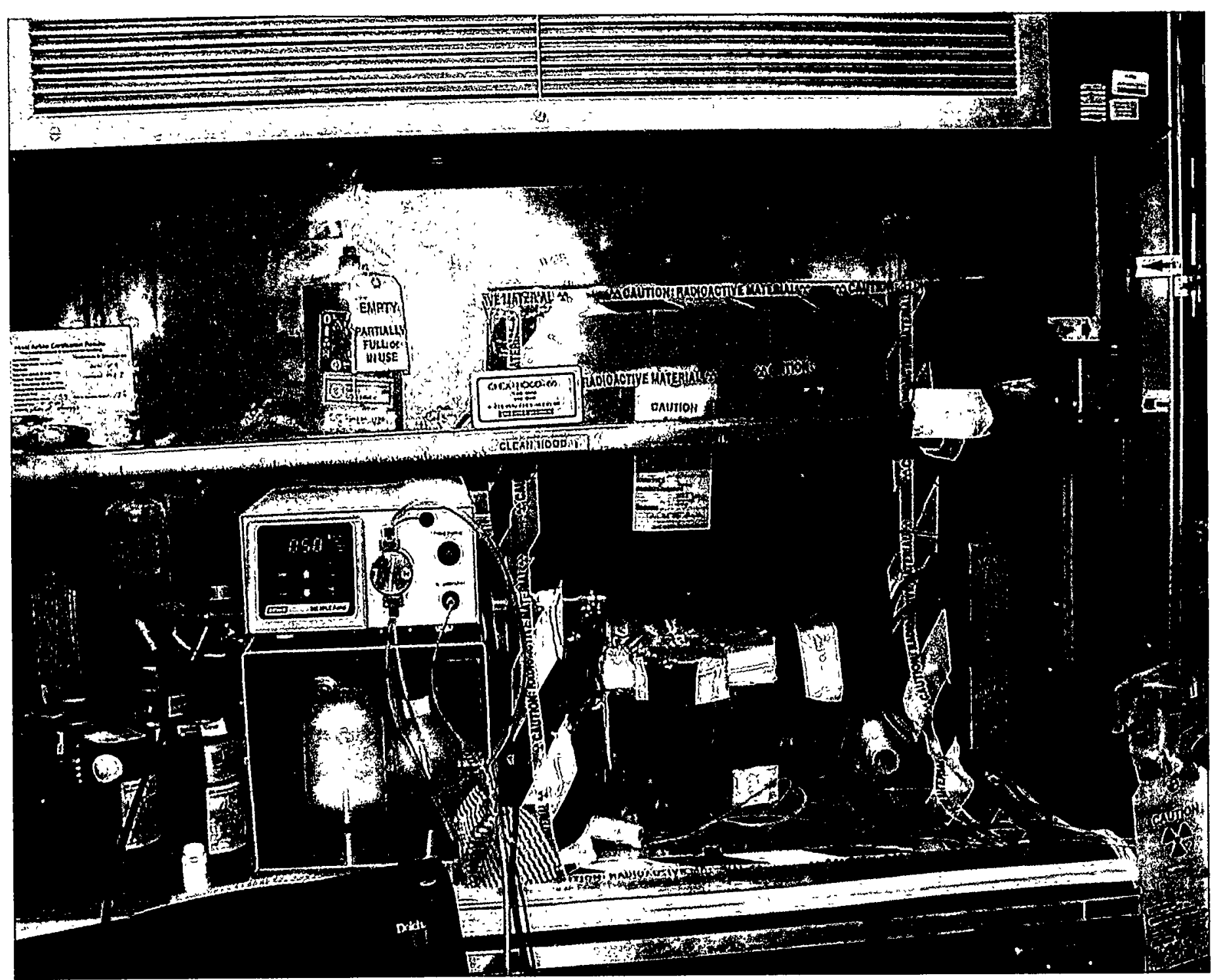




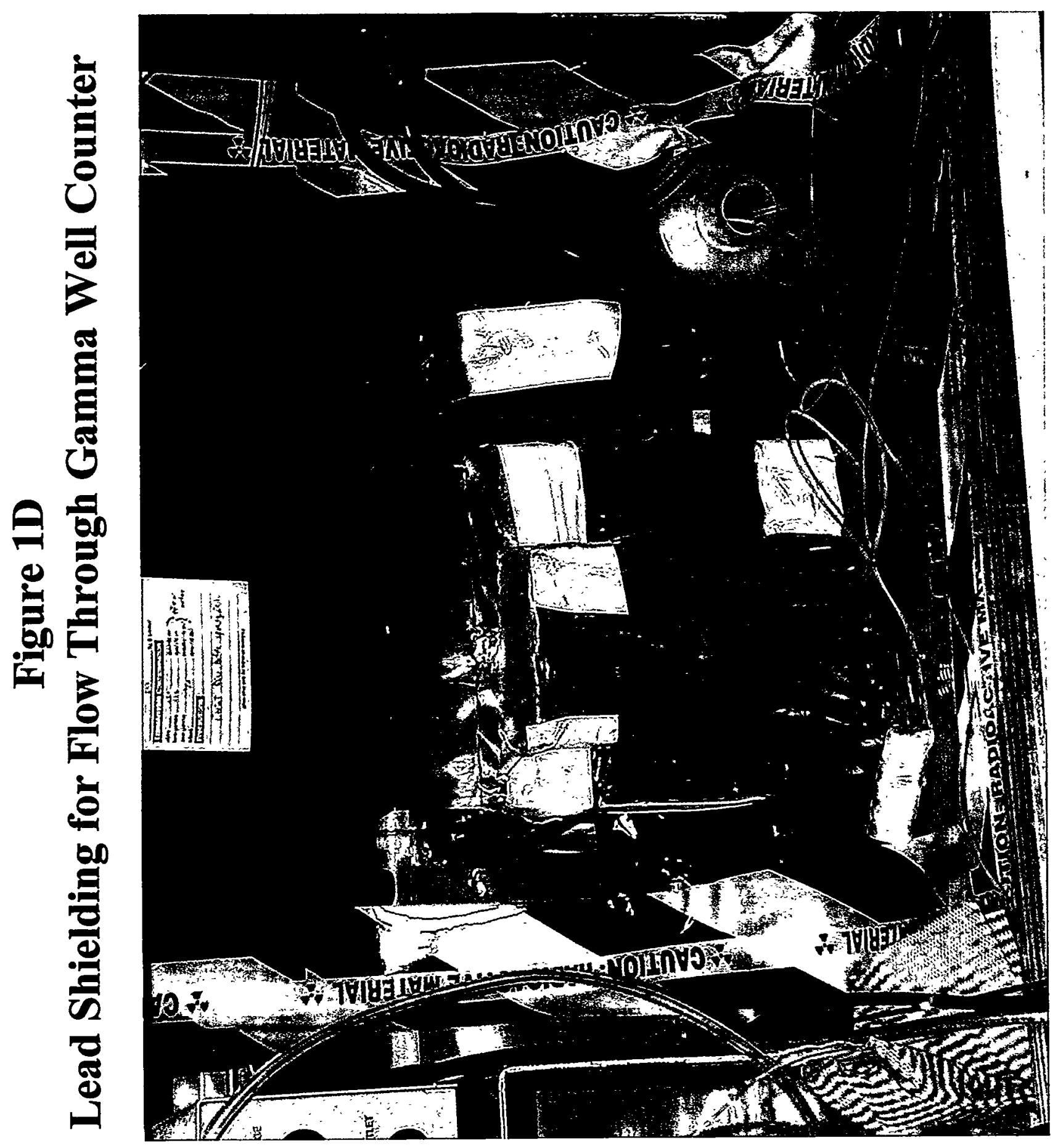




\section{Figure 1E -- HPLC Pump for Nebulizer}

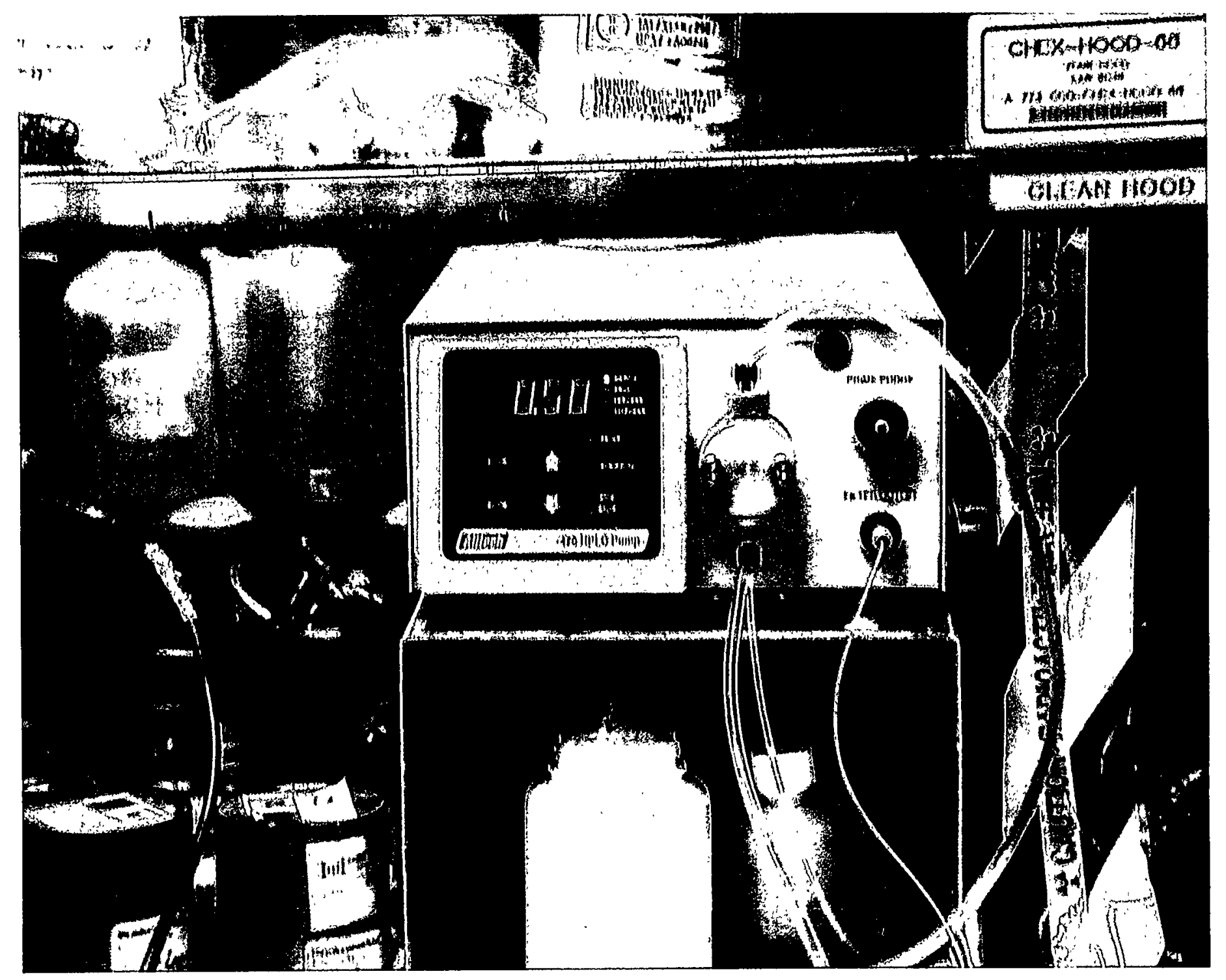




\section{Figure 1F -- Close Up of Cheminert Injection Valve}

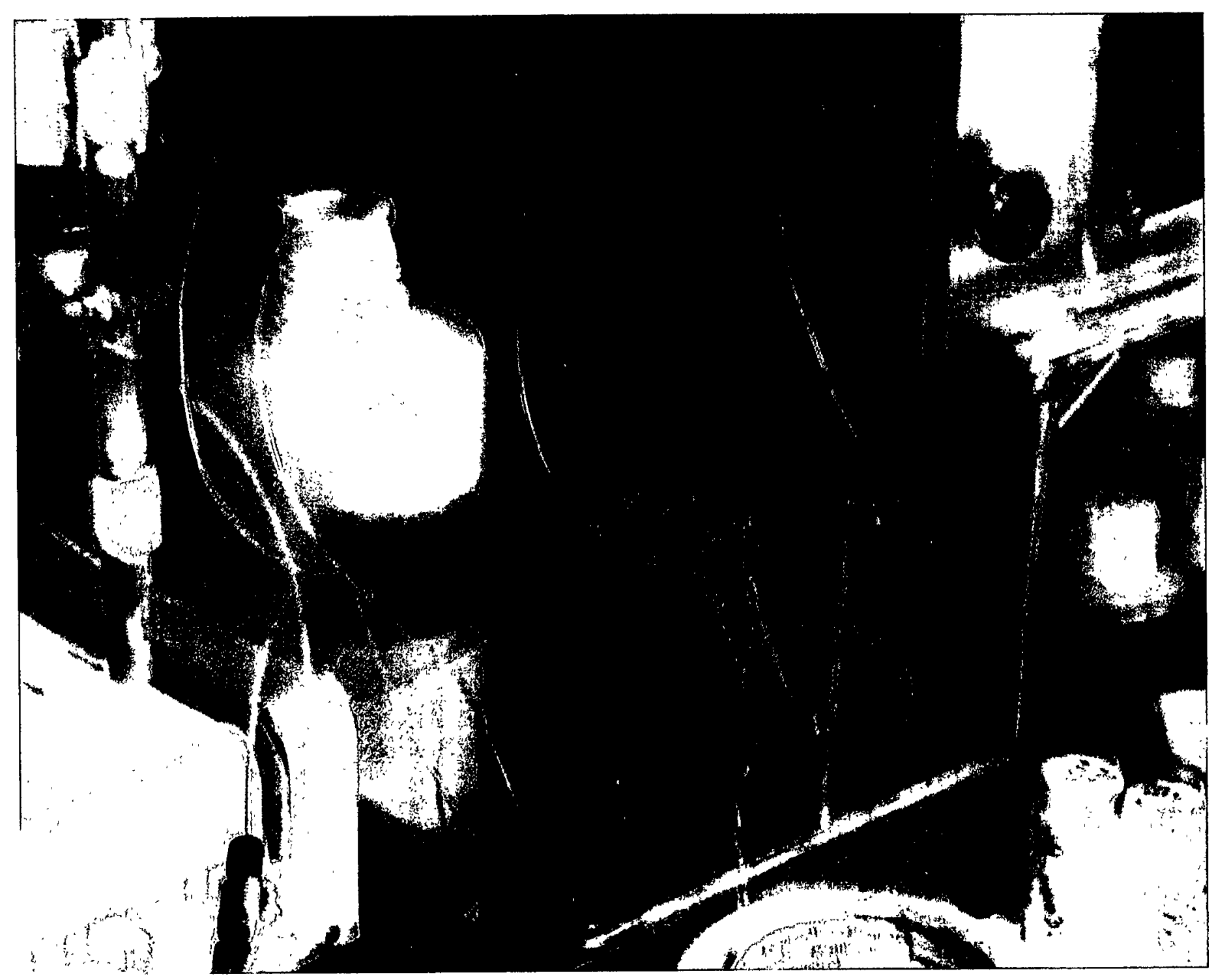




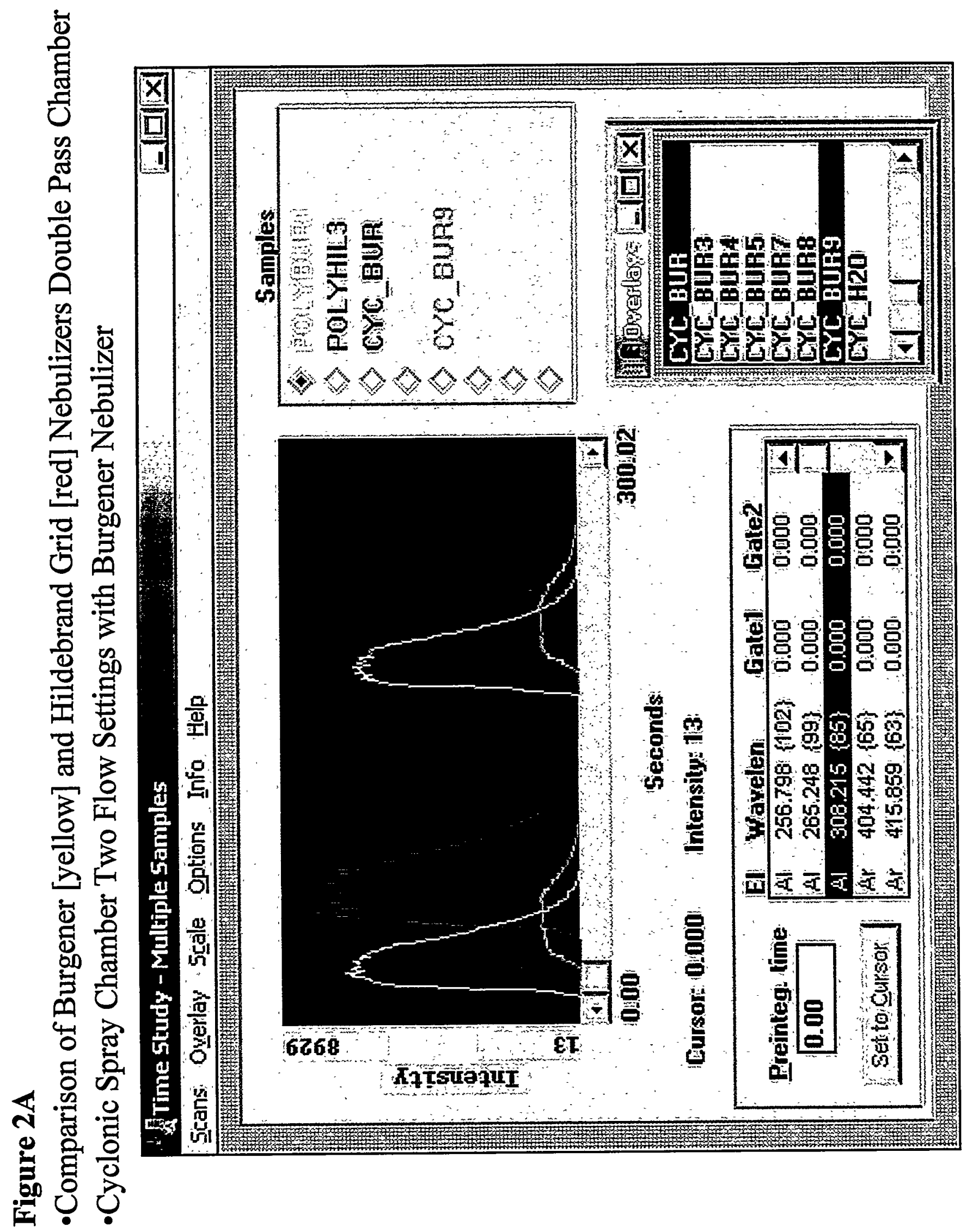




\section{Figure 3A}

\section{Full Spectra Technetium Emissions: $190 \rightarrow 800 \mathrm{~nm}$}

\section{Thermo5PEC/CID Research - [10 ppm Tc - sc blank]}

Image Vlew Map Cursor Options Utilities Window Help

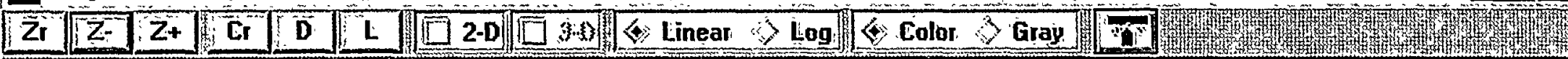

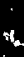

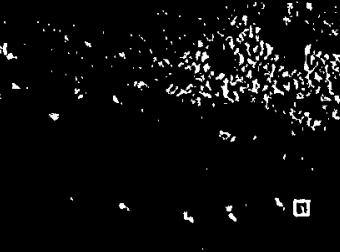

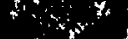
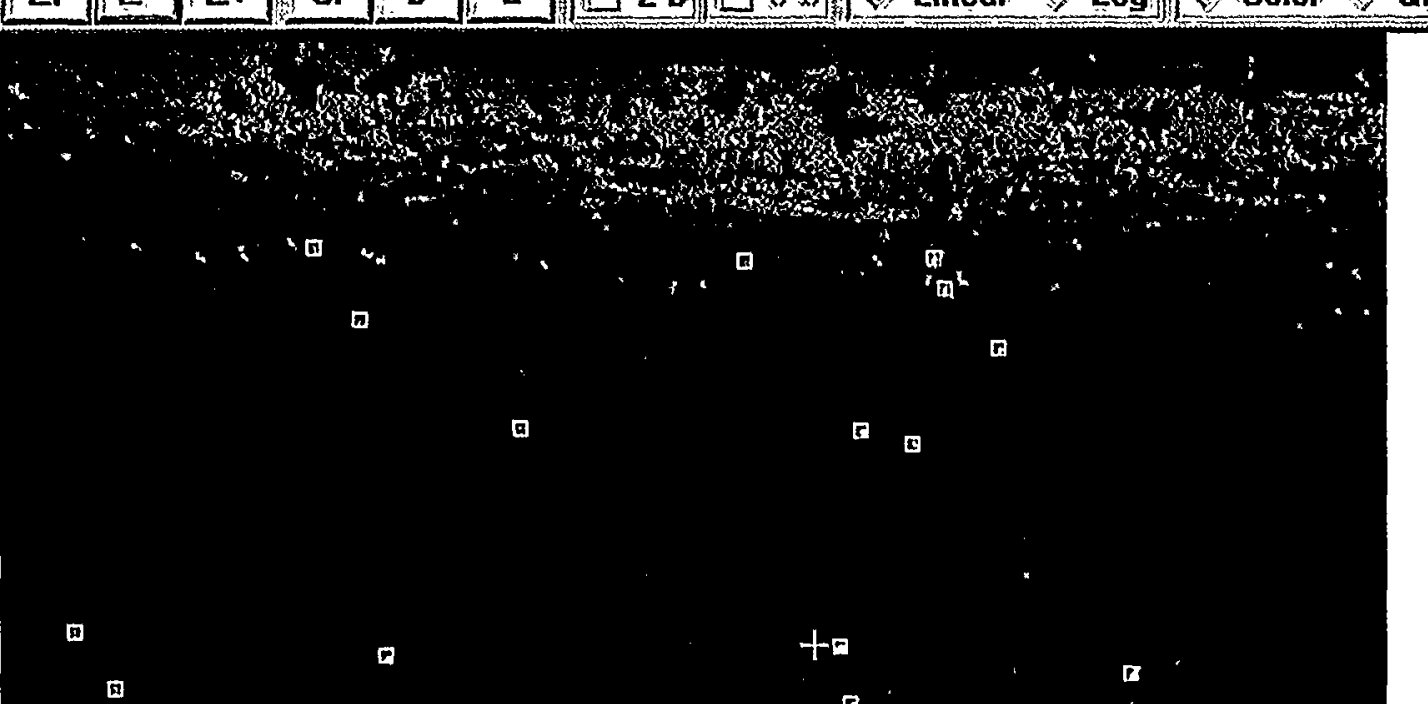

$\mathbf{r} \mathbf{s}$

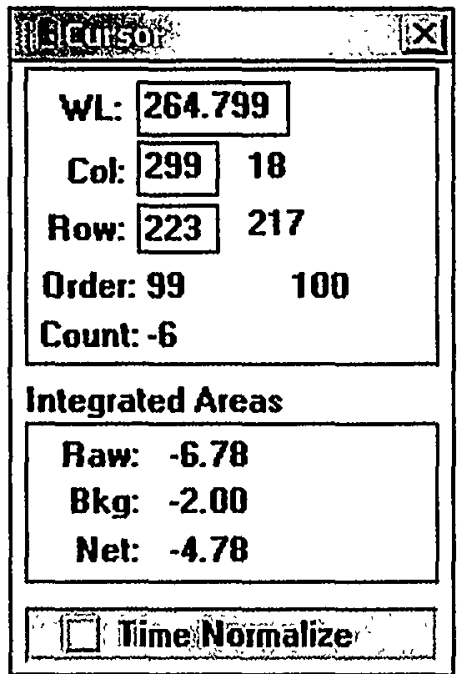

\begin{tabular}{|c|c|}
\hline 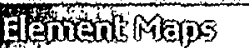 & $\tilde{x}$ \\
\hline Add Maps & 回 Tc \\
\hline$\sqrt{1}$ Draw maps & \\
\hline
\end{tabular}

$+$

5 r

Te

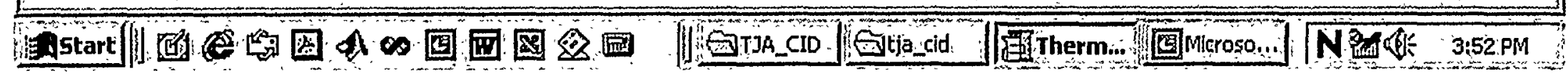




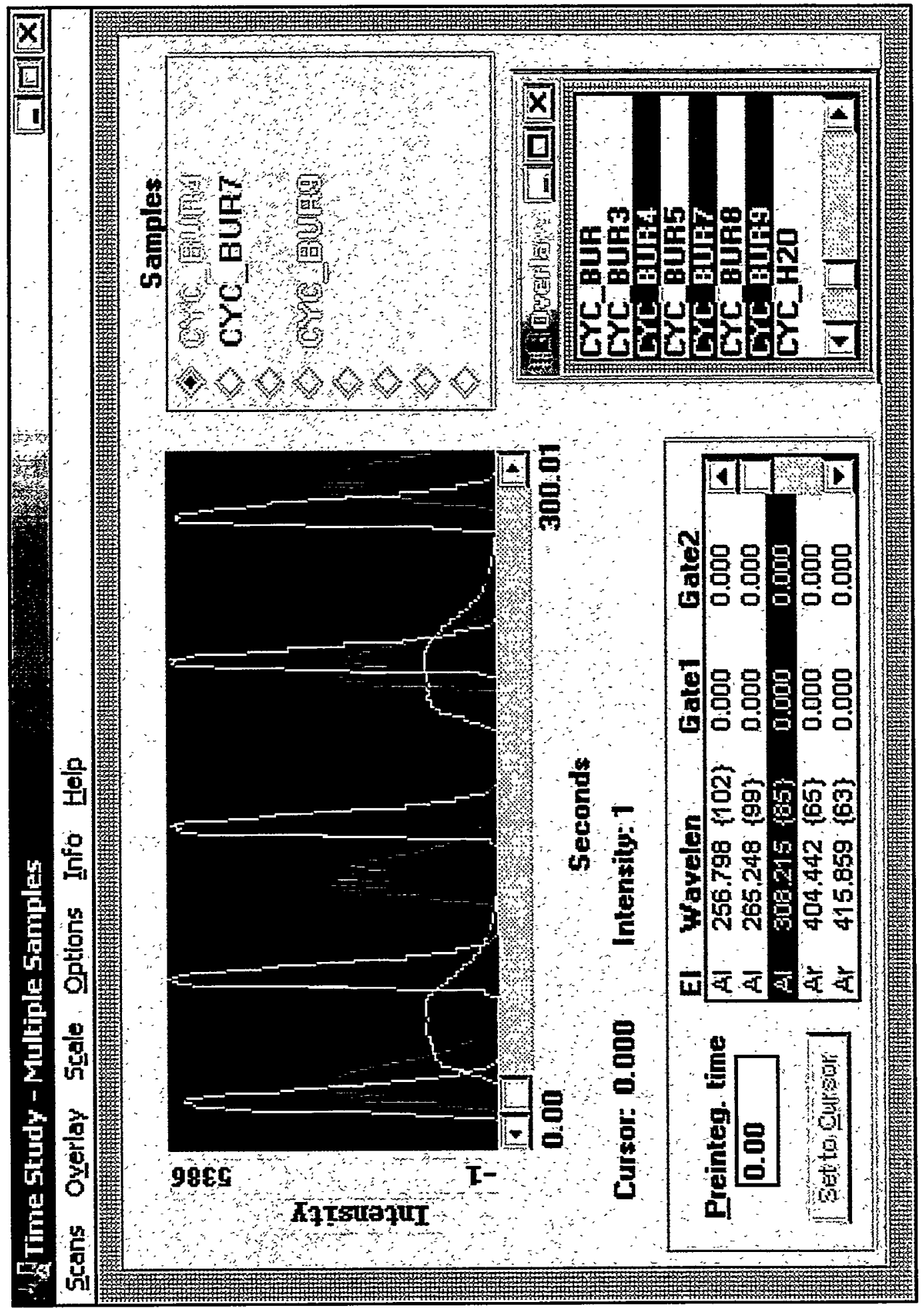




\section{Figure 3B -- Technetium Emission Spectra \\ (Plasma Background Subtracted)}

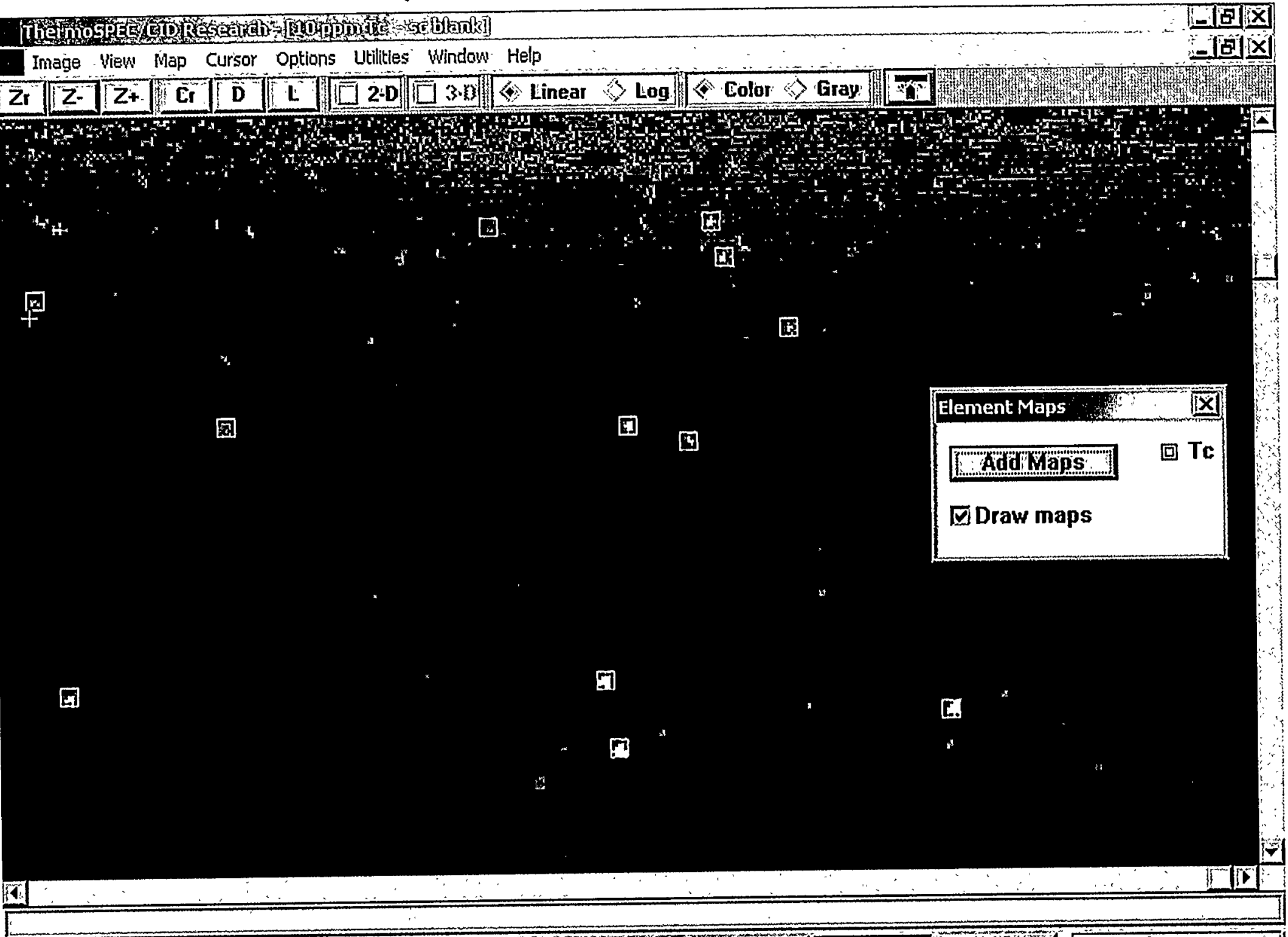

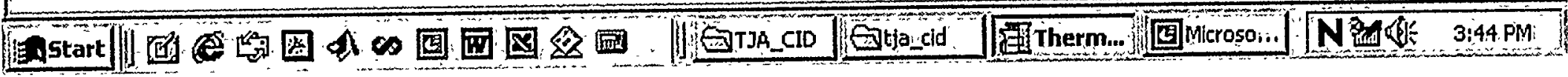


Figure 3C

\section{Close-up Technetium Emissions: $300 \rightarrow 450 \mathrm{~nm}$}

\section{ThermoSPEC/CID Research - [10 ppm Tc - sc blank]}

Image View Map Cursór Options Utilities Window Help

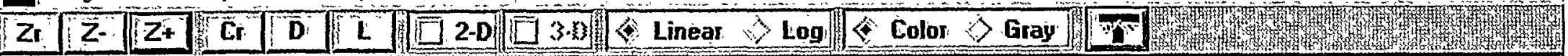

11

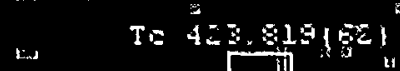

$a$

$n$ r

H.

$\mathfrak{n}_{\pi^{u t}}^{w^{u}}$

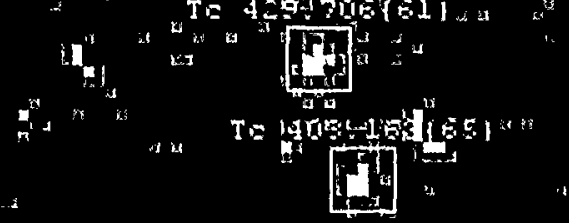

$T=58.0102$

a

$\mathrm{Il}_{\mathrm{a}}$ 


\section{Figure 3D}

\section{Close-up Technetium Emissions: $245 \mathrm{~nm} \rightarrow 330 \mathrm{~nm}$}

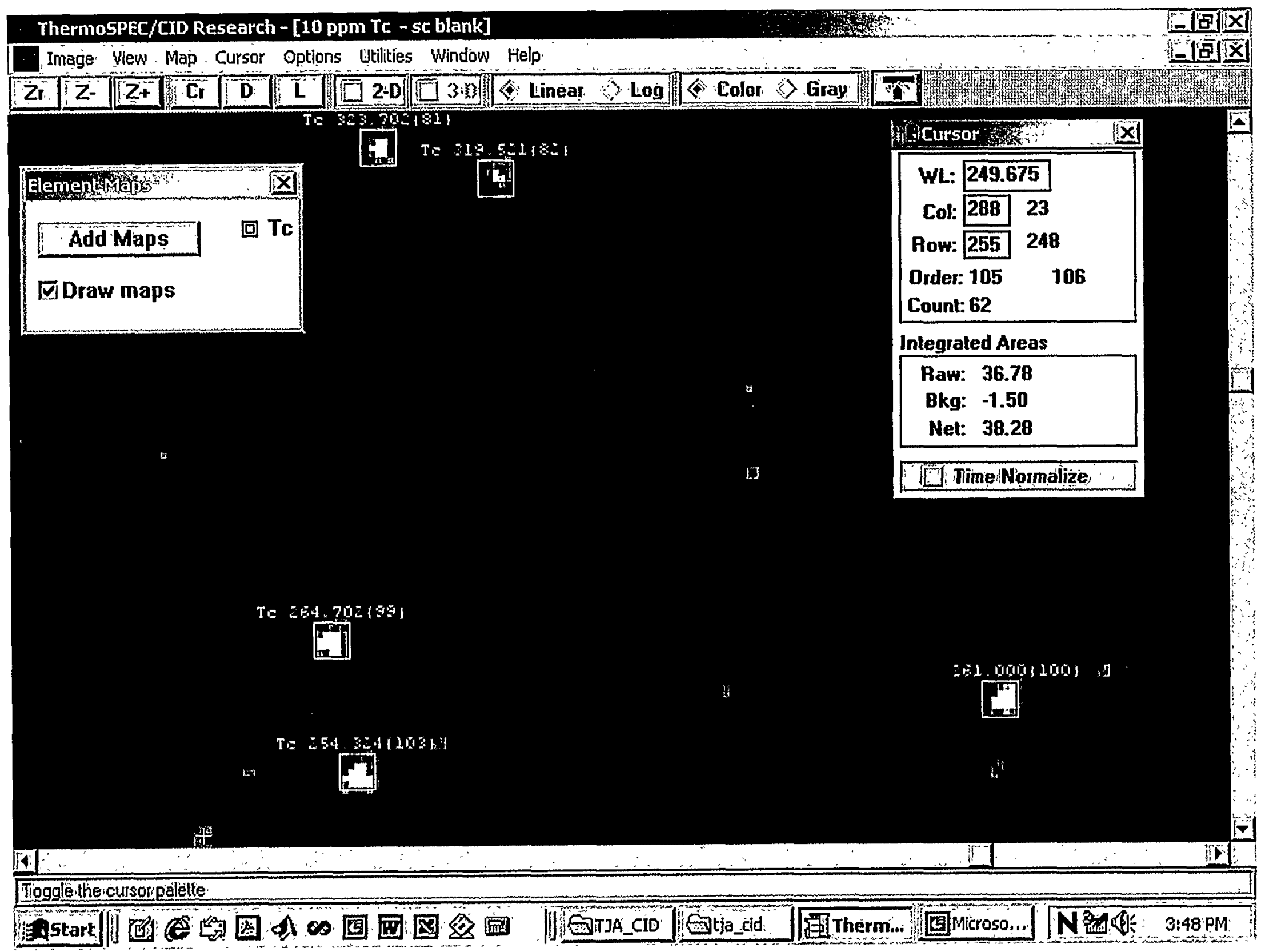


Figure 4A

\section{Effect of Temperature on Elution}
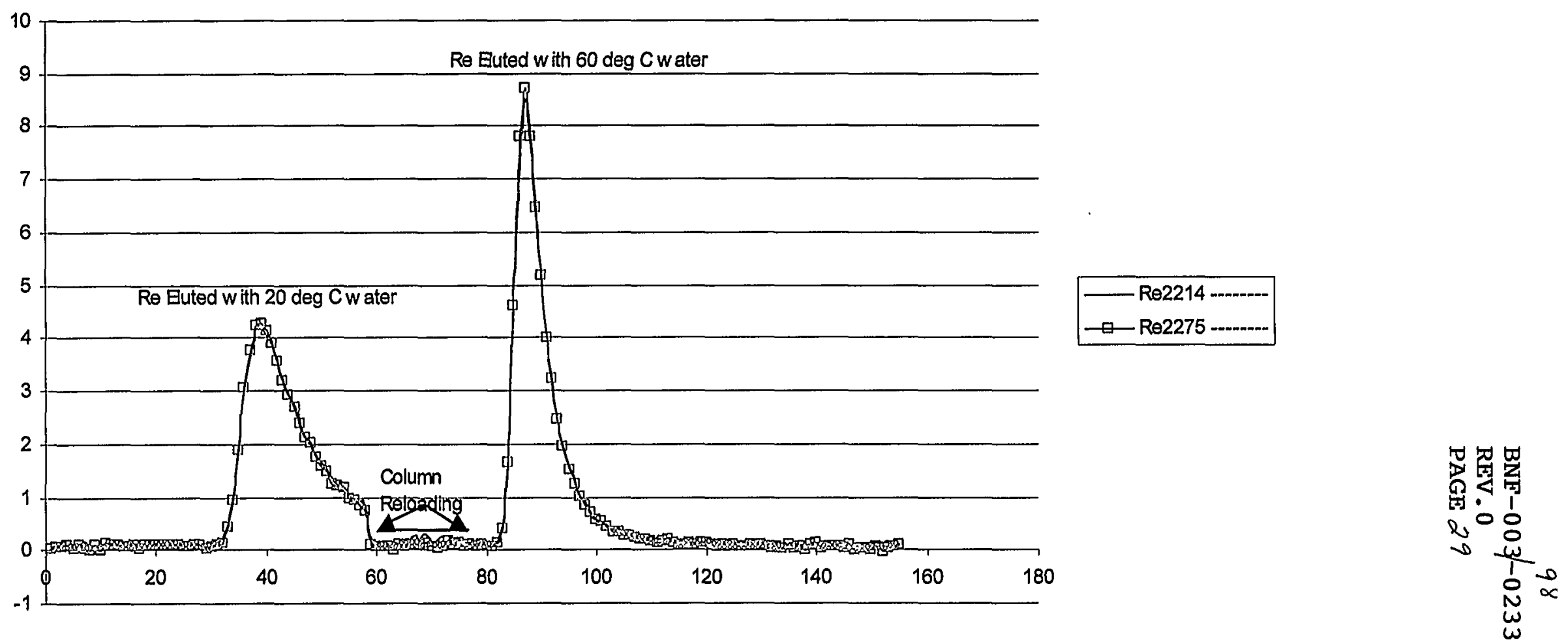
BNF-003/-0233

REV. 0

PAGE 30

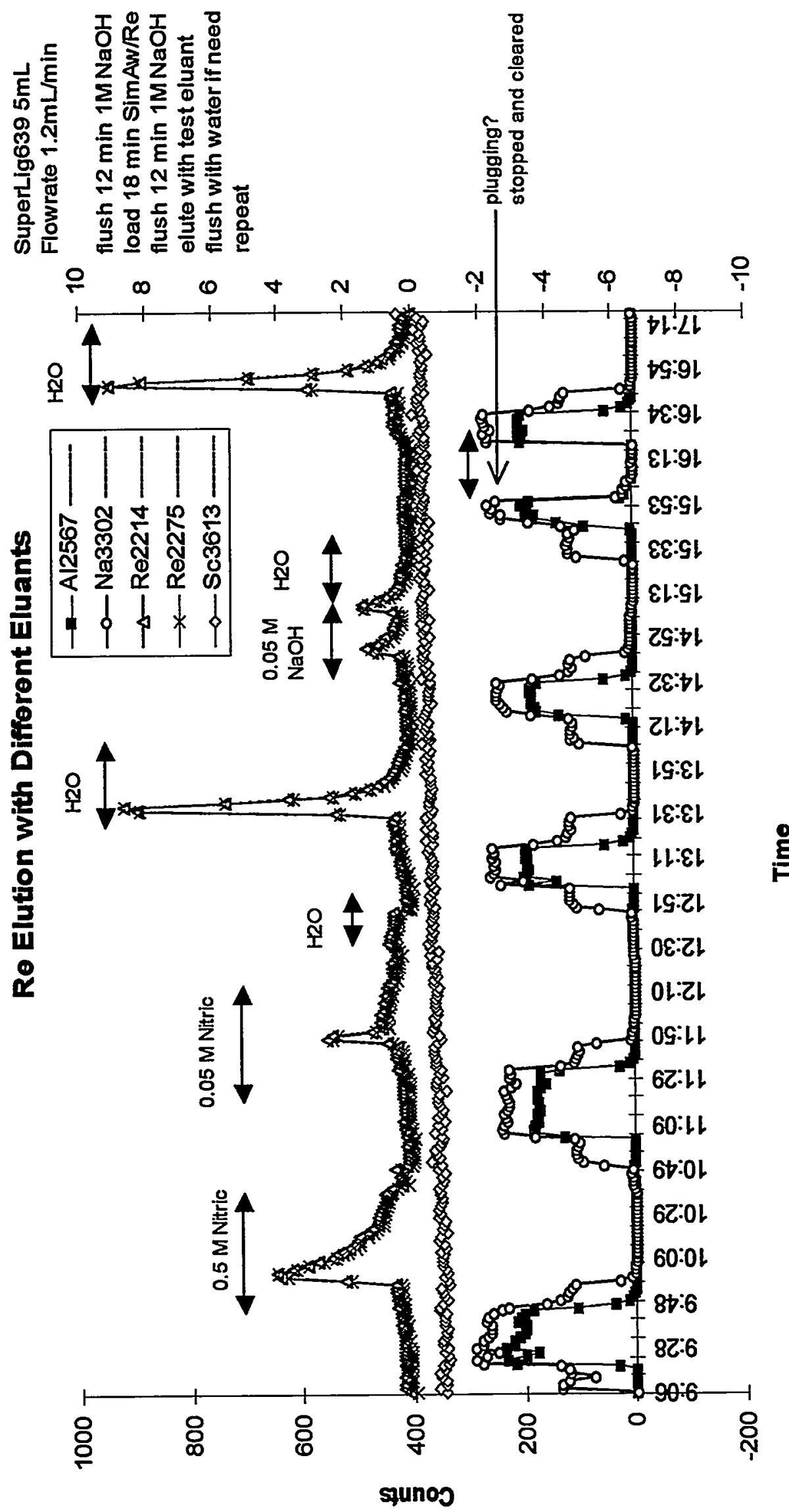




\section{Figure 4B Loading Envelope A For Temperature Run}

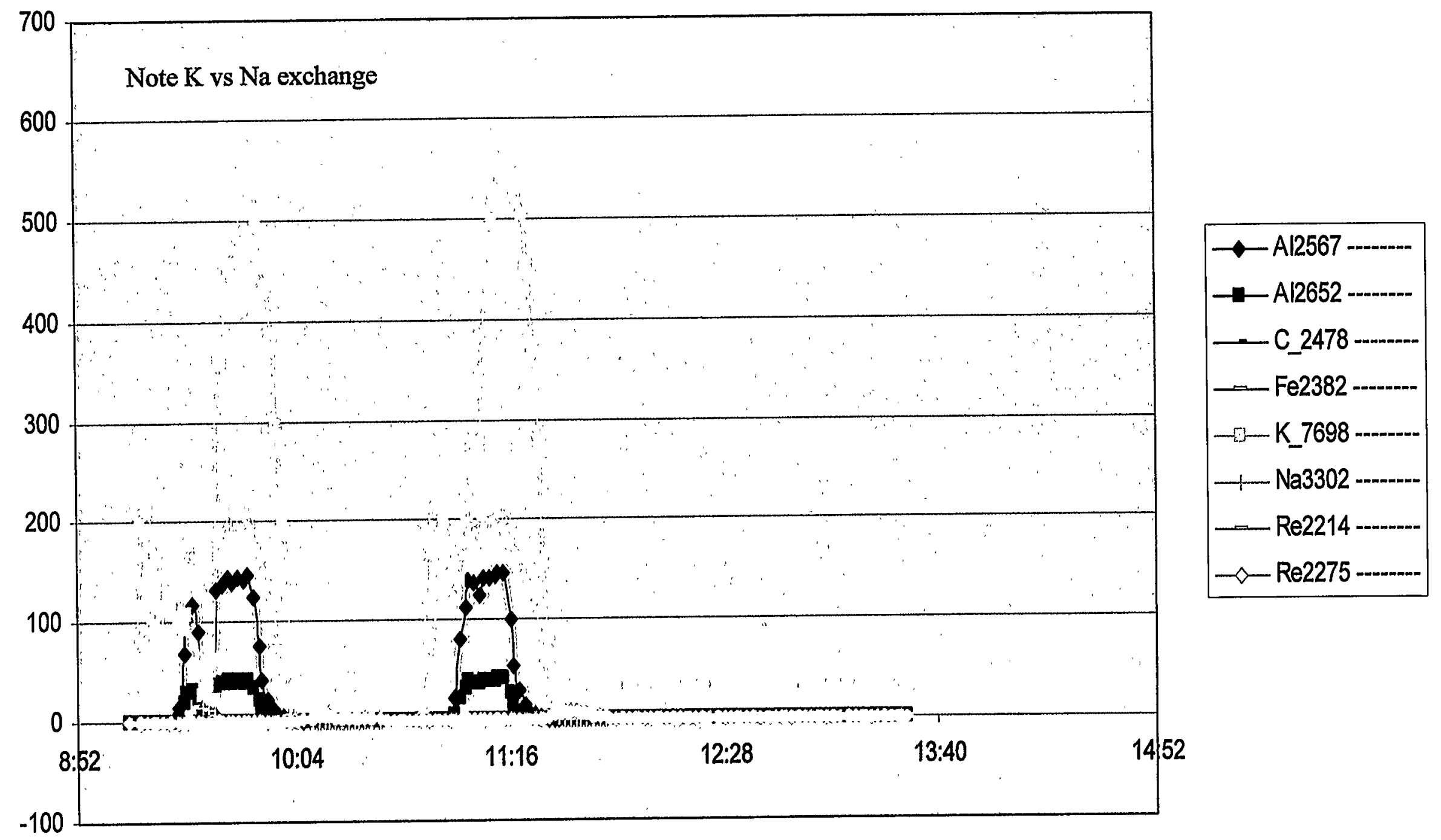

楚皆

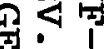

$\omega \circ$ :

$-\frac{1}{1}$ 
98
BNF-0 03/-0233
REV.0
PAGE 32

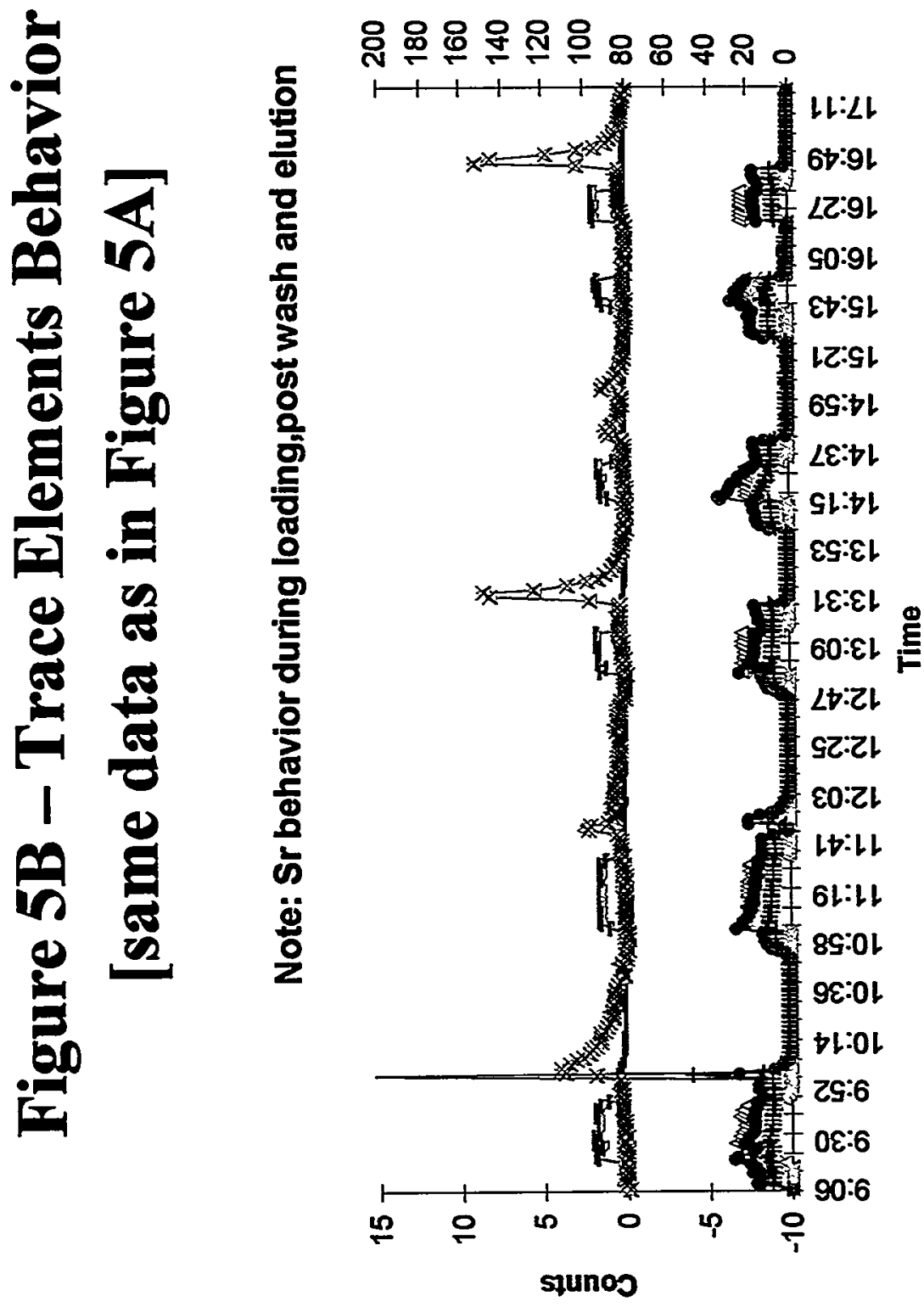


BNF-003/-0233

REV 0

PAGE 33

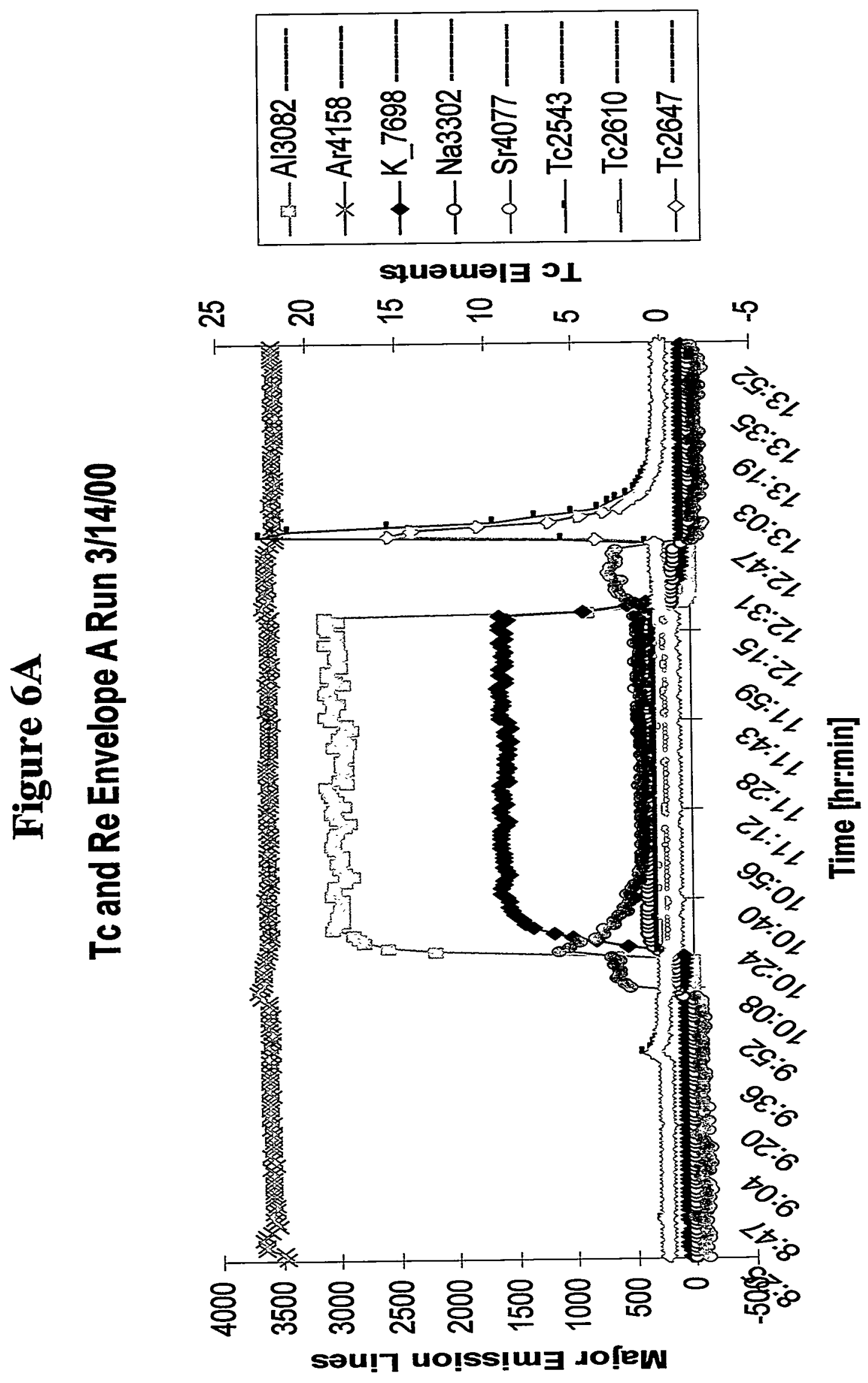




\section{Figure 6B - Te Line Shifts During Loading}

\section{Feed at $\operatorname{Re}[15 \mathrm{ppm}]$ and $\mathrm{Tc}[2.6 \mathrm{ppm}]$ and Eution}

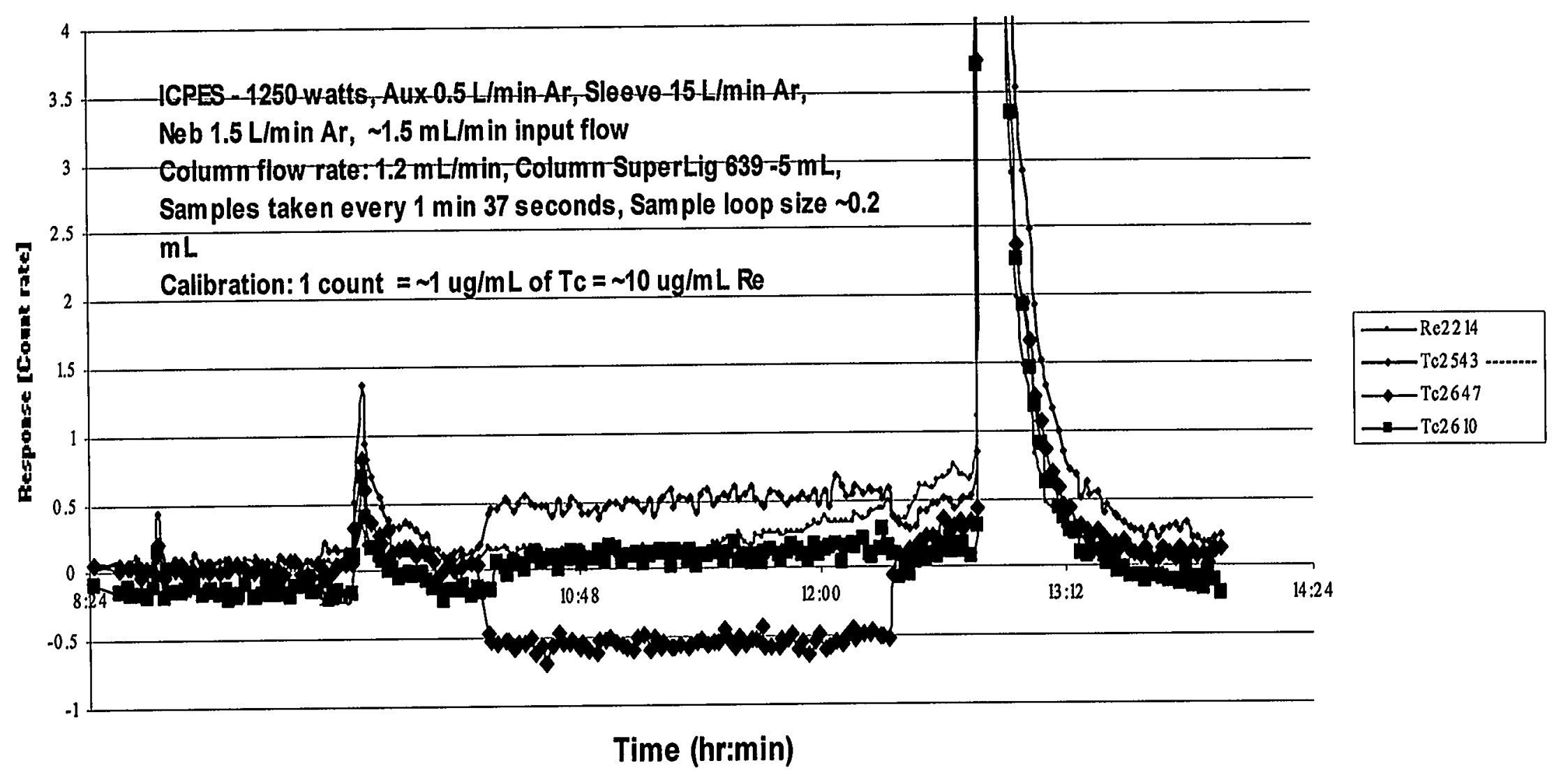



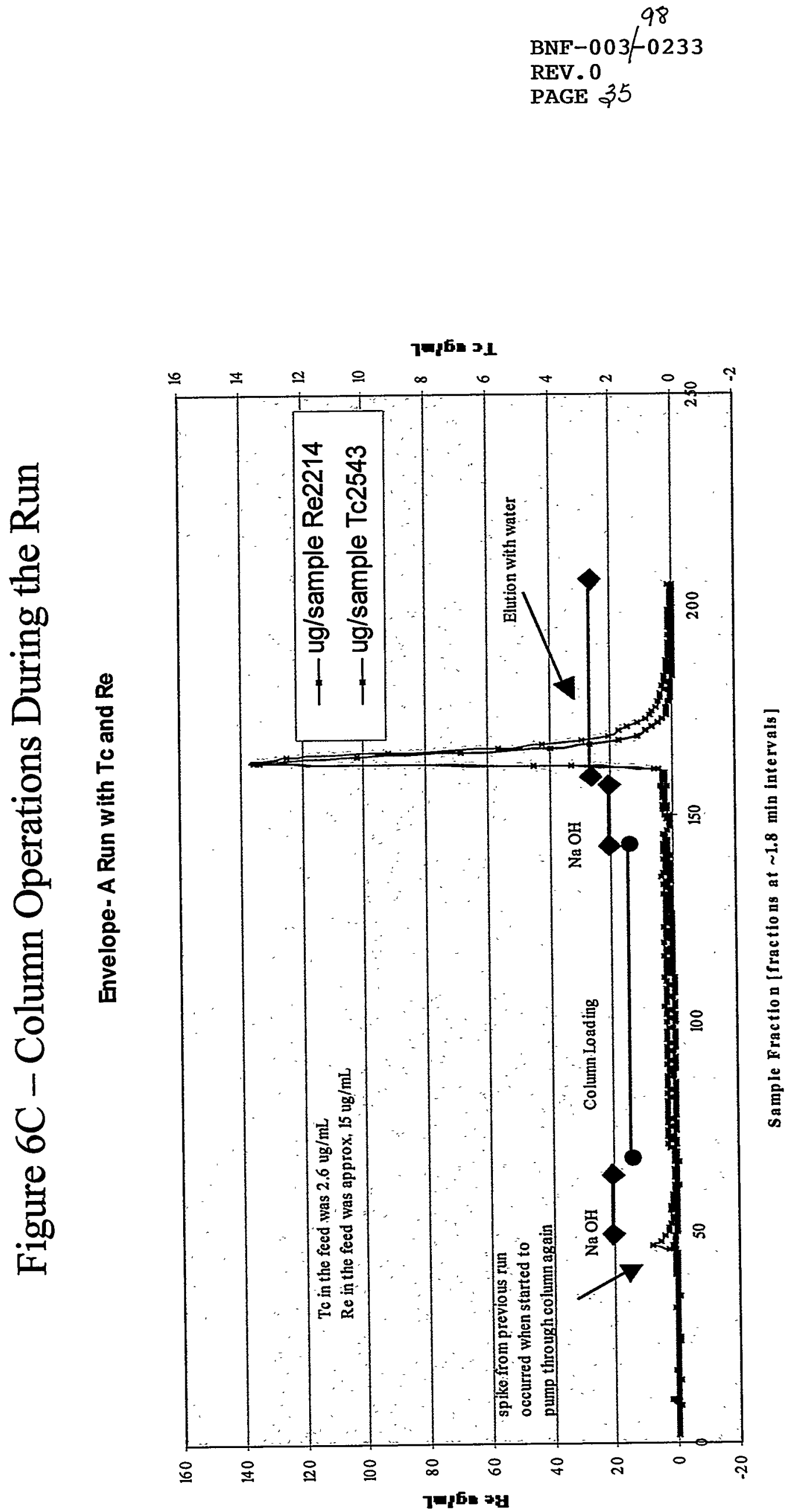

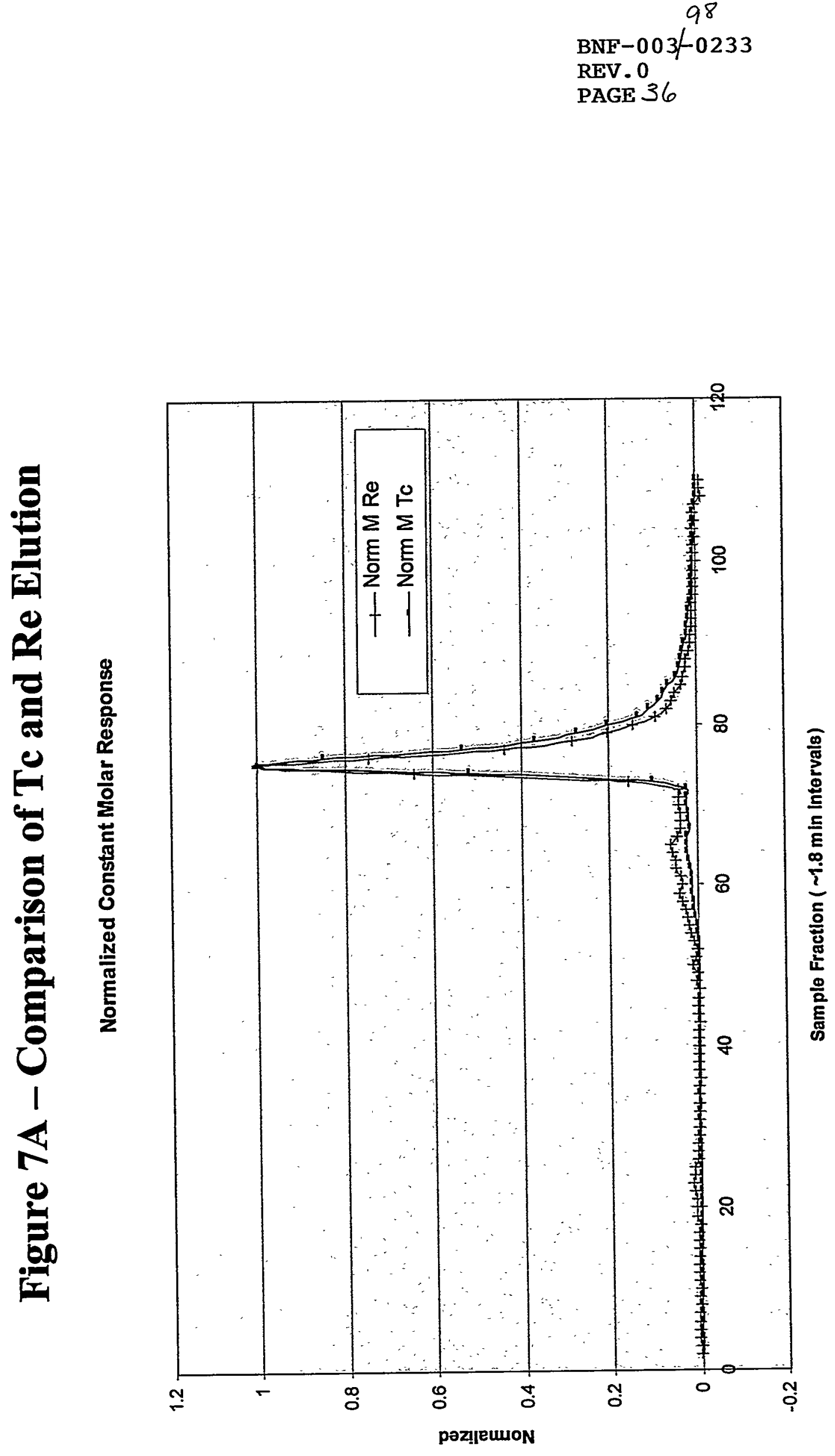

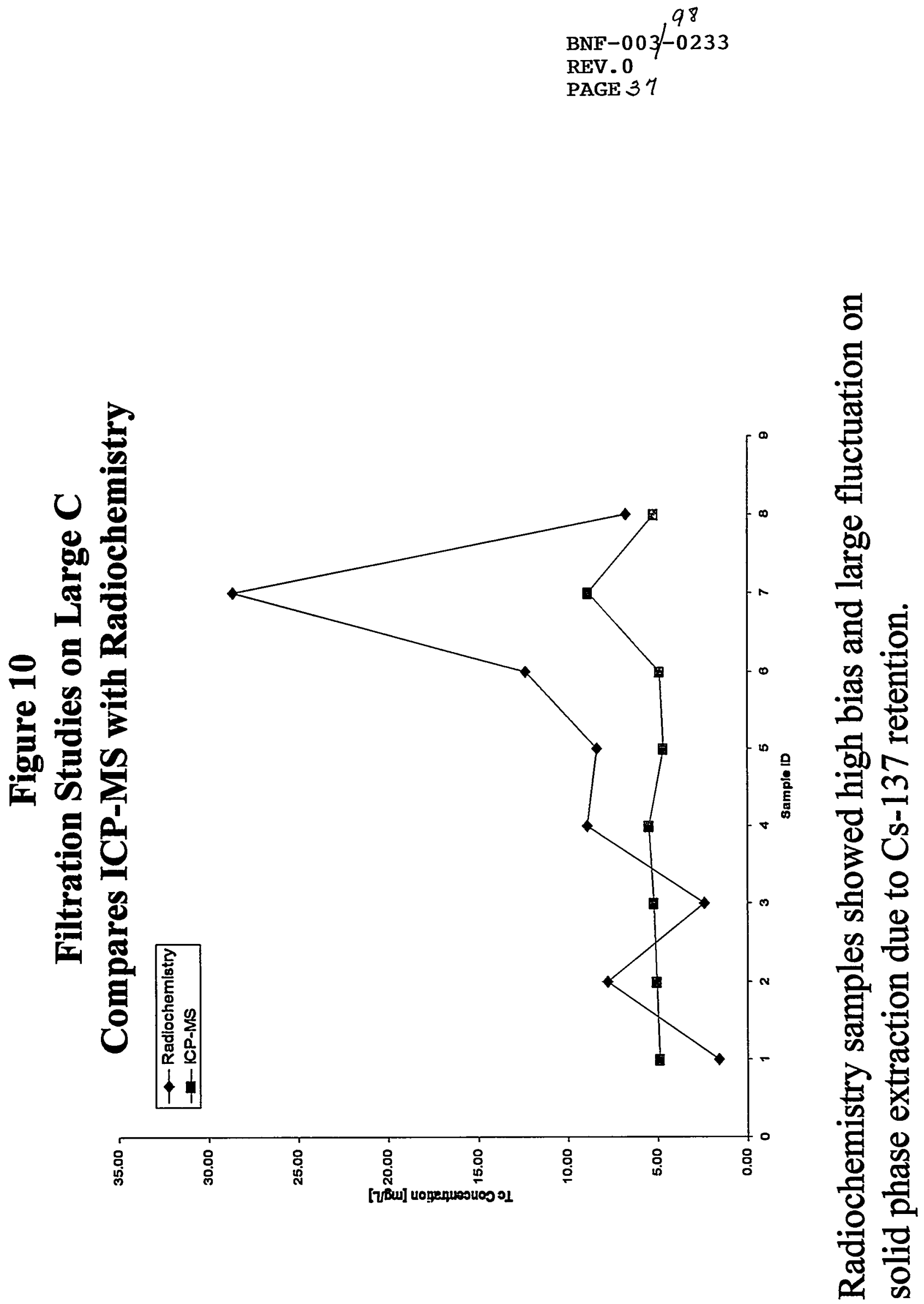


\section{Figure 11 \\ Tc Comparison for Large C : ICP-MS vs. ICP-ES}

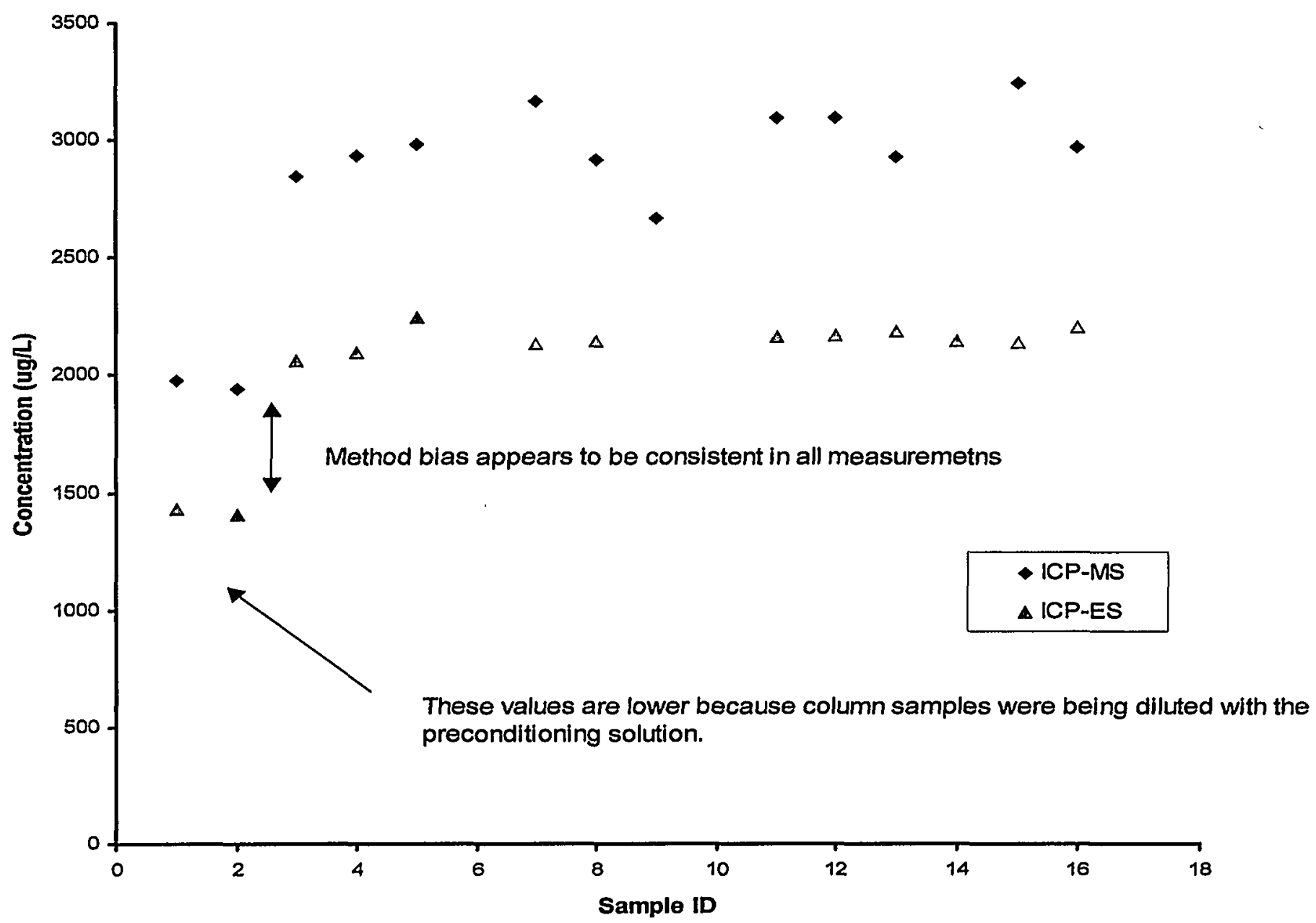


Figure 12

Simulated Envelope C Without Tc Added

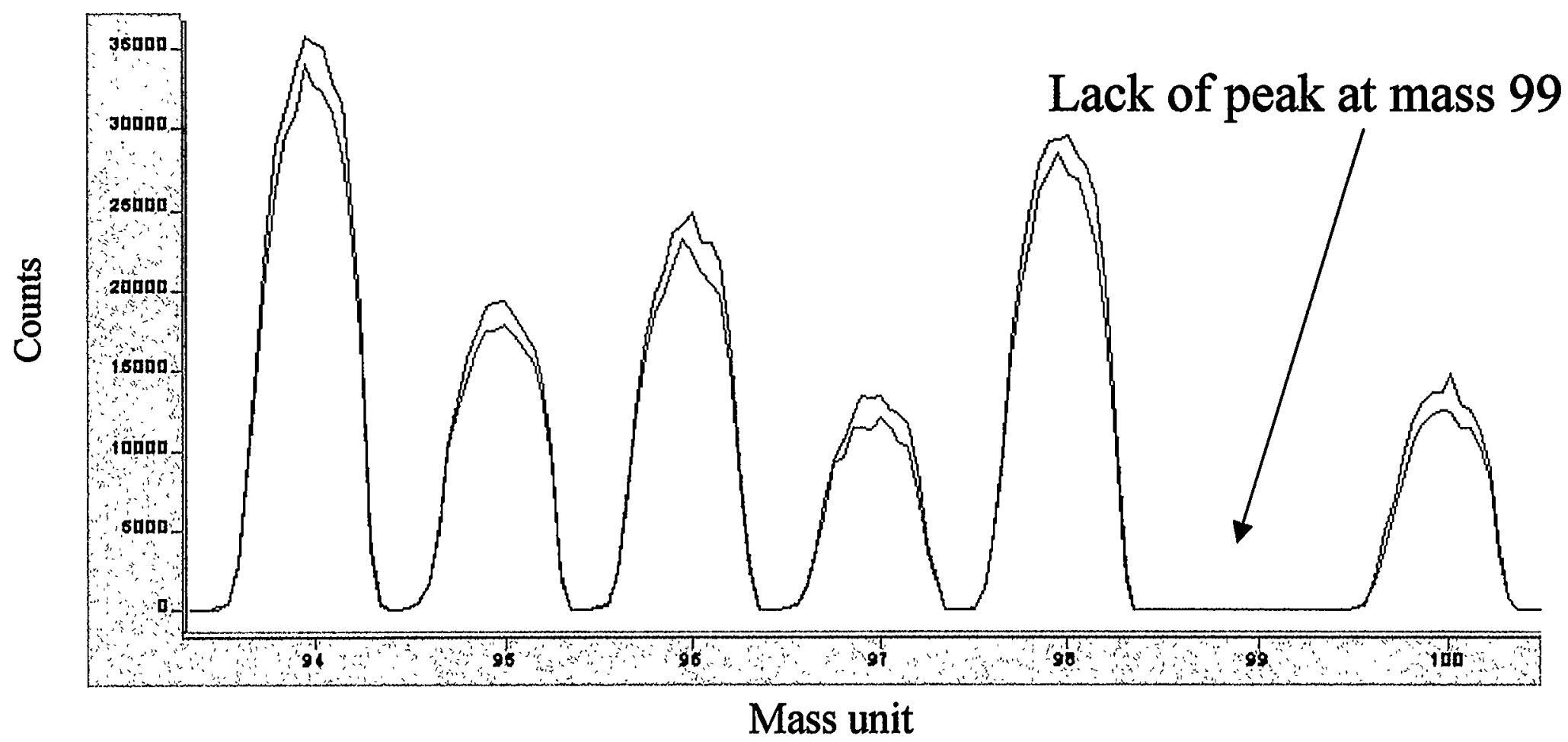

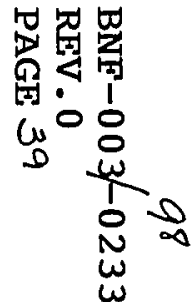

Solution is based on analytical data from tank AN 107 


\section{Figure 13 \\ Decontamination of Envelopes A and B}

\section{Tc in Feed $(\mathrm{mg} / \mathrm{L}) \quad$ Tc in Effluent $(\mathrm{mg} / \mathrm{L})$}
Envelope A
2.4
0.057
Envelope B
14
0.0076

More than $97 \%$ of the Tc in the feed solutions of envelopes $\mathrm{A}$ and $\mathrm{B}$ was removed by the column. If there is an interference at mass 99 in the feed solution, it is less than the error in the method. Only $38 \%$ of the Tc is removed for Envelope C. The majority of Tc is in oxidation states other than 7 for the $\mathrm{C}$ envelope. 
Figure 14

\section{Mass Spectrum of Envelope Large C}

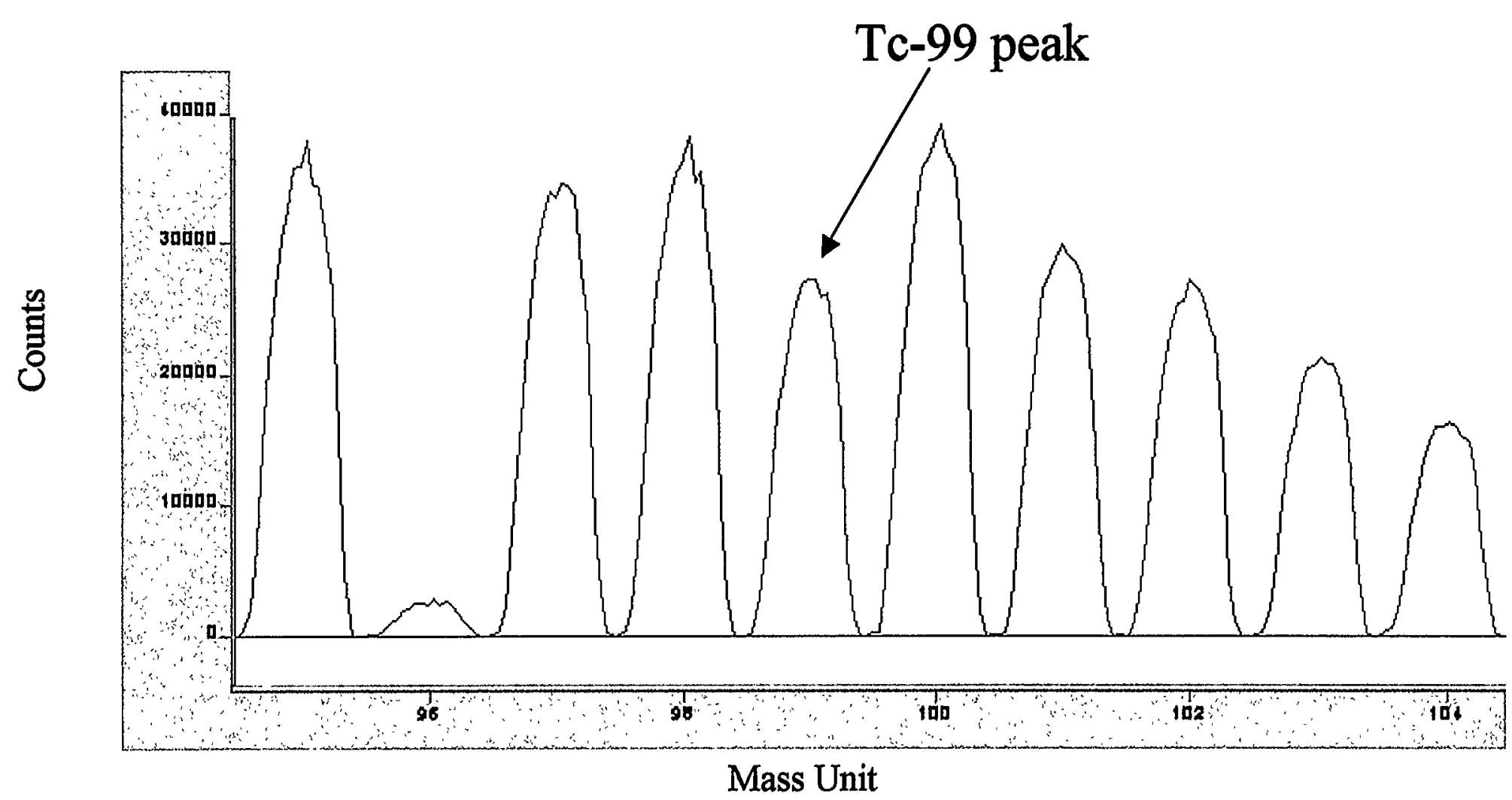

Solution was produced from decontaminated AN 102 


\section{Figure 15}

\section{Measurement of Nearby Isotopes}

During decontamination of Large C, ICP-MS data was collected from 80-247 amu. The results shown below are averaged from 10 samples during decontamination.

$\begin{array}{cc}\text { Mass } & \text { Conc }(\mathrm{mg} / \mathrm{L}) \\ 95 & 6.78 \\ 96 & 0.44 \\ 97 & 6.41 \\ 98 & 6.64 \\ 99 & 3.15 \\ 100 & 7.05 \\ 101 & 5.39 \\ 102 & 4.88 \\ 103 & 3.78 \\ 104 & 2.88\end{array}$

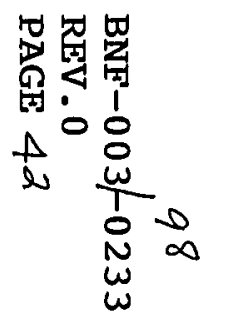


Figure 16

Ru and Mo Isotopic Distributions

\begin{tabular}{cccc} 
Mass & Found & Expected from Fission & \multicolumn{2}{c}{ Expected Natural Distribution } \\
95 & 0.25 & 0.26 & 0.27 \\
97 & 0.24 & 0.24 & 0.16 \\
98 & 0.25 & 0.24 & 0.41 \\
100 & 0.26 & 0.26 & 0.16 \\
101 & 0.41 & 0.46 & 0.25 \\
102 & 0.37 & 0.38 & 0.47 \\
104 & 0.22 & 0.16 & 0.28
\end{tabular}

Molybdenum and Ruthenium exhibit fission product isotopic distributions. Mass 104 is most likely a combination of $\mathrm{Ru}$ and SrO. It is unlikely that there is a large $\mathrm{SrC}$ interference because of the good match at ${ }^{100} \mathrm{Mo}$. 


\title{
BNF-003/-0233 \\ REV. 0 \\ PAGE 44
}

Quantitative Measurement of Chromium, Manganese, Rhenium, and Magnesium in Liquid by Laser-induced Breakdown Spectroscopy

Fang-Yu Yueh, Ramesh C. Sharma, Jagdish P. Singh, and Hansheng Zhang Diagnostic Instrumentation and Analysis Laboratory

Mississippi State University

Grant No. Task order GA0046

\author{
Final Report \\ Submitted to \\ Dr. William Spencer \\ Savannah River Technology Center \\ Savannah River Site \\ Aiken, South Carolina 29808
}

May 29, 2000 


$$
\begin{aligned}
& \text { BNF-003/-0233 } \\
& \text { REV.0 } \\
& \text { PAGE } 45
\end{aligned}
$$

\section{TABLE OF CONTENTS}

\section{TABLE OF CONTENTS}

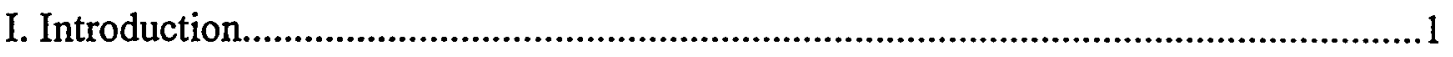

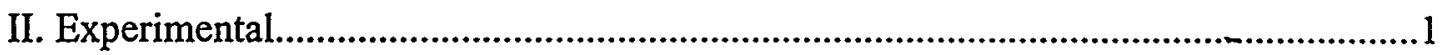

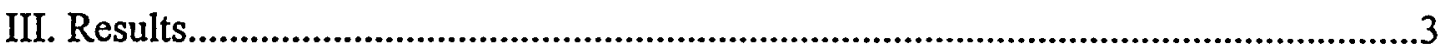

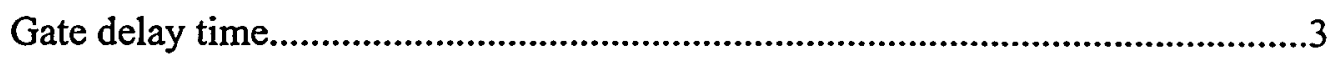

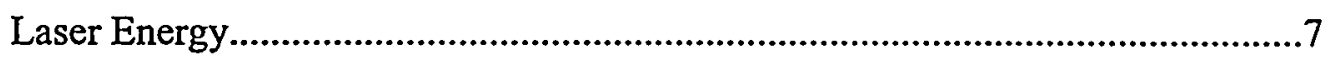

Lens-to-surface distance....................................................................................

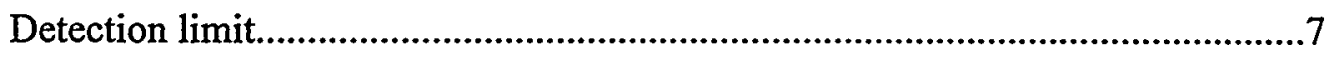

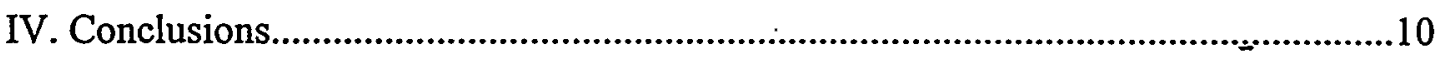

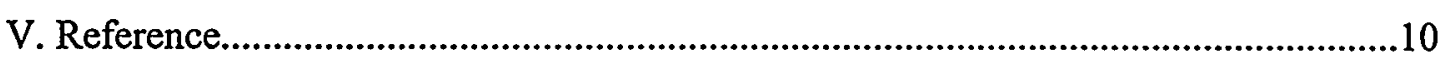




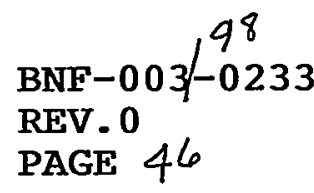

\section{INTRODUCTION}

British Nuclear Fuels Limited (BNFL), Inc. provides low activity and high level waste processing service at the Department of Energy (DOE) Hanford site. High level wastes will be processed as a pretreatment step prior to vitrification. The wastes are run through an ion exchange column to remove Tc from Hanford supernate tank waste. The effluent from the removal column goes to one type of radioactive glass and the stripped technetium goes to another higher activity glass. An on-line Tc monitor is required to monitor the column effluent to assure that technetium is sent to the correct process streams. The required technique has to be able to measure Tc below the $100-\mu \mathrm{g} / \mathrm{L}$ level. It should achieve at least $10 \%$ confidence interval at 1000 $\mu \mathrm{g} / \mathrm{L}$.

Laser induced breakdown spectroscopy (LIBS) is a laser-based, non-intrusive, and sensitive optical diagnostic technique for measuring the concentration of various atomic and molecular species in test media. ${ }^{1,2}$ It uses a high power laser beam to produce a laser-induced plasma at the test point. The plasma atomizes and electronically excites the various atomic species present in the test volume in a single step. The intensities of the atomic emission lines observed in the LIBS spectrum are used to infer the concentration of the atomic species. LIBS has been successfully demonstrated its real-time monitoring capability in various field tests. ${ }^{3-8}$ It uses gated detection to discriminate the strong plasma continuum background emission. It can provide a real-time measurement of several of the most critical metals. The major thrust of this work is to evaluate the analytical figures of merit of LIBS system for Tc measurement. The results of this study will be used to determine the feasibility of using LIBS as a Tc monitor at the DOE Hanford site.

In this work, current DIAL's LIBS system was optimized for detection of $\mathrm{Cr}, \mathrm{Mg}, \mathrm{Mn}$ and $\mathrm{Re}$ in liquid. $\mathrm{Cr}, \mathrm{Mg}$, and $\mathrm{Mn}$ have similar properties to technetium. $\mathrm{Mg}$ is chosen because some $\mathrm{Mg}$ lines are in the same spectral region as the most sensitive $\mathrm{Tc}$ lines. So we can evaluate the system sensitivity near the Tc emission lines. Various optical geometries which produce the laser spark in and at the liquid sample were tested to determine the best geometry for liquid measurement. The optimized experimental condition were determined and used to determine the . limit of detection (LOD) of these four test elements. The results of the study is summarized below.

\section{EXPERIMENTAL}

The detail experimental setup of the DIAL LIBS system is described in reference 3 and is shown in Figure 1. The output $(1064 \mathrm{~nm})$ of a Nd:YAG laser was frequency doubled to $532 \mathrm{~nm}$ using a doubling crystal. A dichroic mirror was used to separate the frequency doubled output from its fundamental frequency. An ultraviolet (UV) grade quartz lens was used to focus the laser beam. The same lens was used to collect light from the laser-induced spark. Two UV-grade quartz lenses of focal lengths $100 \mathrm{~mm}$ and $50 \mathrm{~mm}$ were used to couple the LIBS signal to an optical fiber bundle. The other end of the fiber bundle was coupled to an optical spectrograph, whose entrance slit was replaced by the rectangular exit end of the optical fiber. An intensified charge coupled device (ICCD) detector attached to the exit slit of the spectrograph was used to detect the light from the laser spark. The detector was operated in a gated mode to record LIBS signal. To maximize the signal, the gate delay time and gate width were adjusted for each 


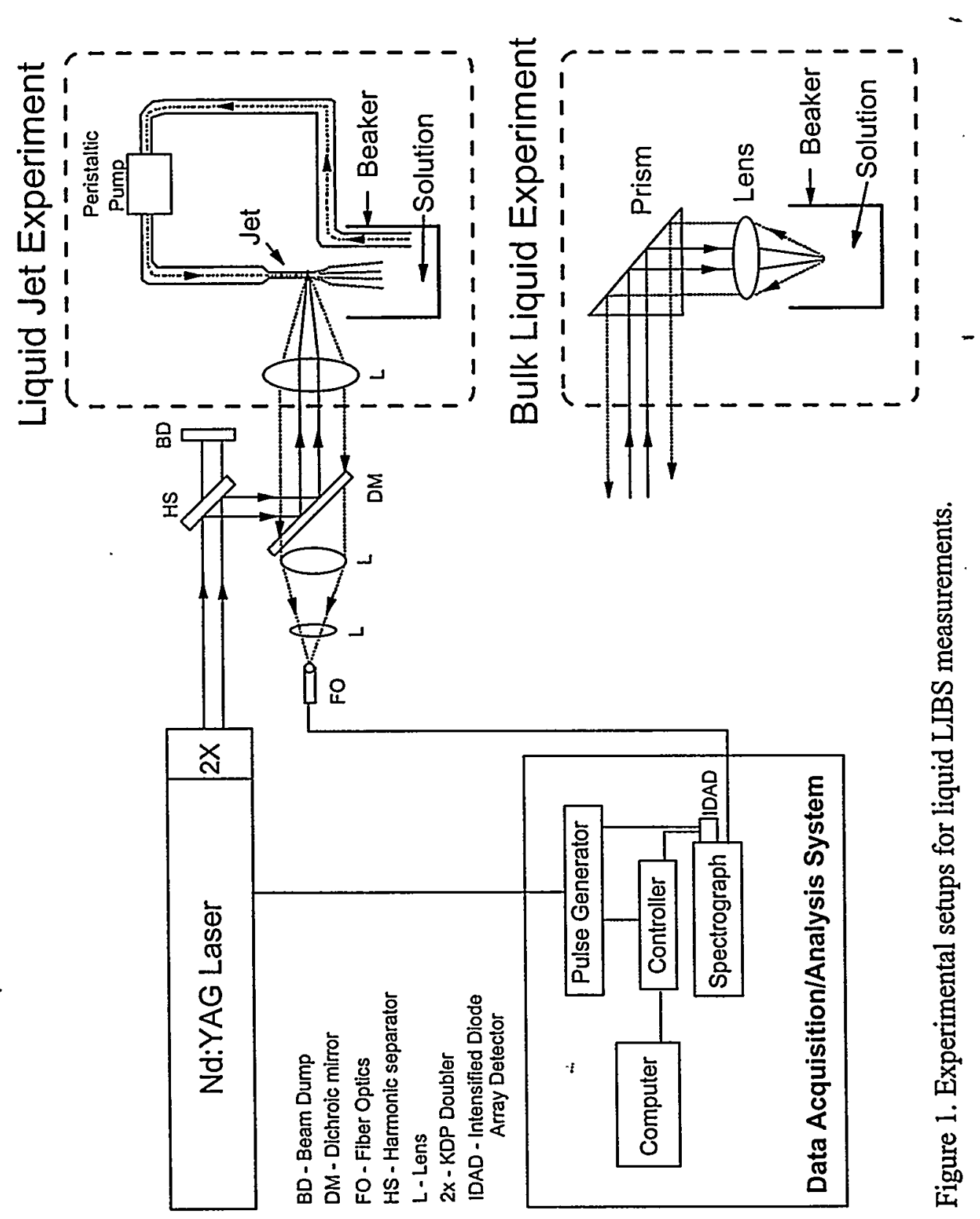


element to achieve the best signal-to-noise ratio data. The spectrograph is equipped with two grating $2400 \mathrm{l} / \mathrm{mm}$ and $3600 \mathrm{l} / \mathrm{mm}$, respectively. LIBS data were recorded with both grating to determine which grating give the best detection limit in a specified spectral region. Data acquisition and analysis were performed using a personal computer.

To determine the best experimental setup for solution measurement, the performance of two experimental setups (see Figure 1) were evaluated. The solution used in the study was prepared by diluting the ICP standard from AccuTrace in distilled water. In the first experiment, the sample solution was kept in a small beaker and laser beam was focused on the liquid surface to provide the measurement from the bulk sample. The scatter light by the splashed liquid droplet was properly blocked. The LIBS signal from the bulk liquid is collected in the backward direction. In the second experiment, a liquid jet system was assembled, to provide a stable solution jet. The liquid sample in a bottle was pumped by a peristaltic pump (Cole-Parmer Instrument Co.) through a tubing of 5-mm internal diameter (ID) to a custom-made nozzle. It produced a steady jet of $1.5-\mathrm{mm}$ diameter. The laser beam was focused from the direction perpendicular to the liquid jet and LIBS signals were collected in backward direction.

\section{RESULTS}

The survey spectra of $\mathrm{Cr}, \mathrm{Mg}, \mathrm{Mn}$, and $\mathrm{Re}$ were recorded to determine the most sensitive lines for this study. Table I lists the most sensitive lines of these elements found. The experimental parameters which can affect LIBS detection limit most are gate delay time, laser energy, and lensto-surface distance. These parameters are carefully studied for both bulk liquid and liquid jet and the results are summarized below.

\begin{tabular}{|l|l|}
\hline Element & Atomic lines \\
\hline $\mathrm{Mn}$ & $403.076 \mathrm{~nm}, 403.307 \mathrm{~nm}, 403.449 \mathrm{~nm}$ \\
\hline $\mathrm{Mg}$ & $279.553 \mathrm{~nm}^{*}, 280.27 \mathrm{~nm} *, 285.2 \mathrm{~nm}$ \\
\hline $\mathrm{Cr}$ & $425.44 \mathrm{~nm}, 427.48 \mathrm{~nm}, 428.972 \mathrm{~nm}$ \\
\hline $\mathrm{Re}$ & $346.046 \mathrm{~nm}, 346.473 \mathrm{~nm}$ \\
\hline
\end{tabular}

* Ion lines

\section{Gate delay time}

To optimize LIBS signal, LIBS data were recorded at different laser energies and detection windows. Since the continuum background and atomic emission decay with different rates, it is possible to obtain an optimum detection window far each element. Figure 2 shows the variation of atomic line signal and background signal with time. The continuum background are dominated in the first several microseconds and decay much faster than the analyte signal at later time. By adjusting the gate delay time, LIBS data with best signal-to-background ratio can be recorded. Figure 3 shows LIBS spectra of Mg recorded at different delay times in bulk liquid and liquid jet. It is clear that a shorter delay time is required for the bulk liquid measurement than the liquid jet measurement for reasonable $\mathrm{S} / \mathrm{N}$ ratio. 
BNF-003 $\begin{gathered}98 \\ -0233\end{gathered}$

REV. 0

PAGE 49

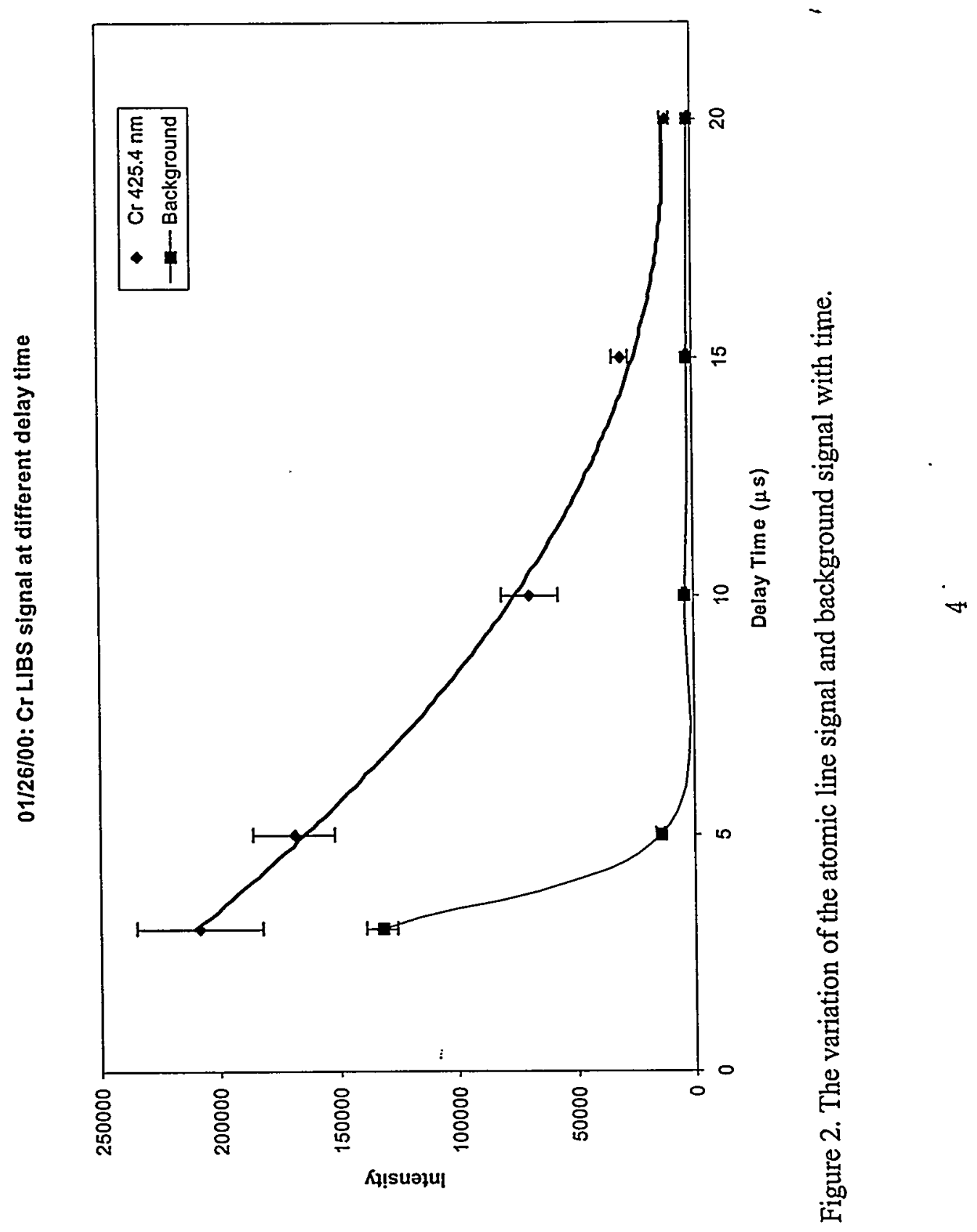


BNF-003 $J^{98}-0233$

REV. 0

PAGE 50
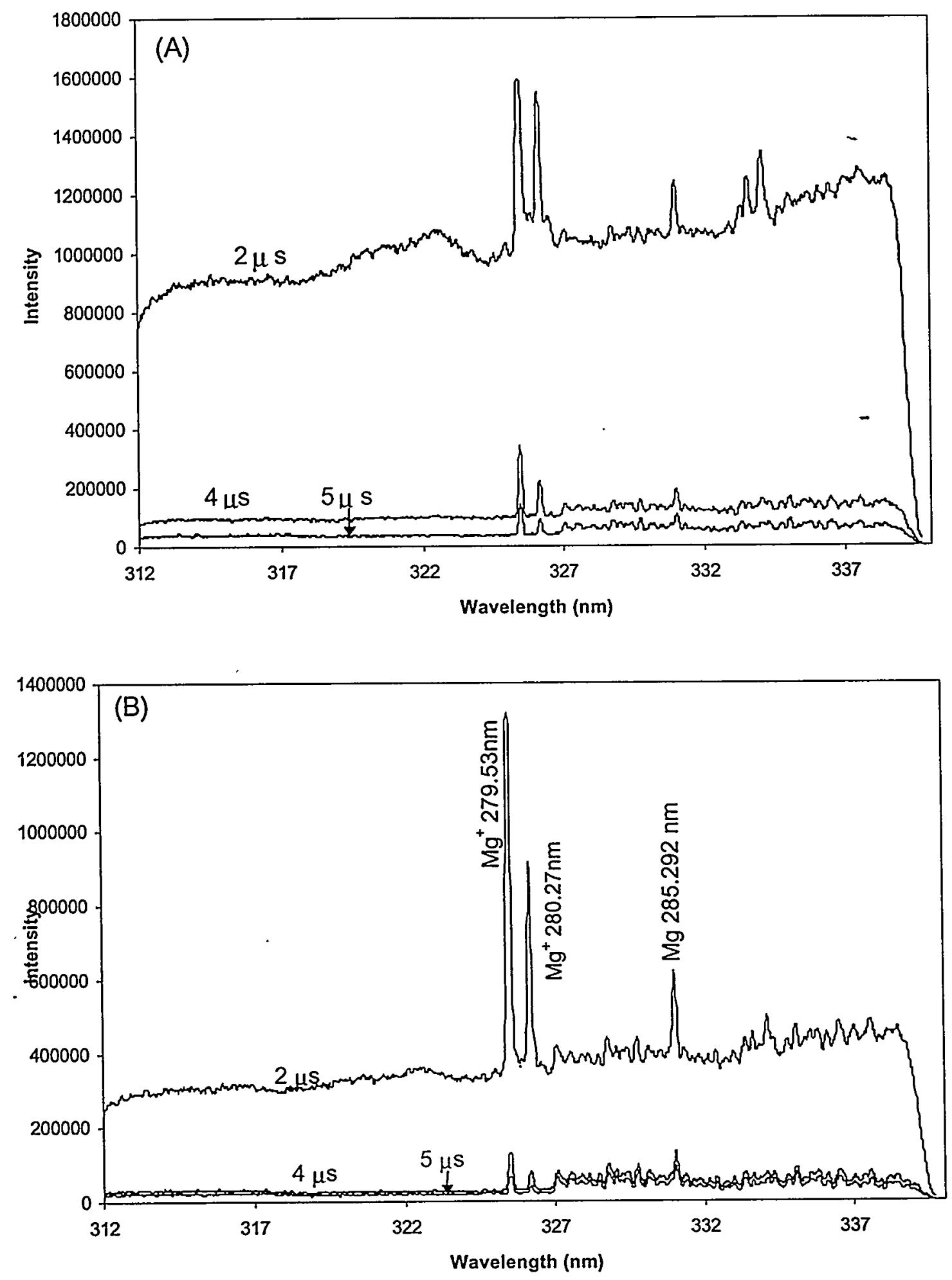

Figure 3. LIBS spectra of $\mathrm{Mg}$ recorded at different delay times in (A) bulk liquid and (B) liquid jet. 


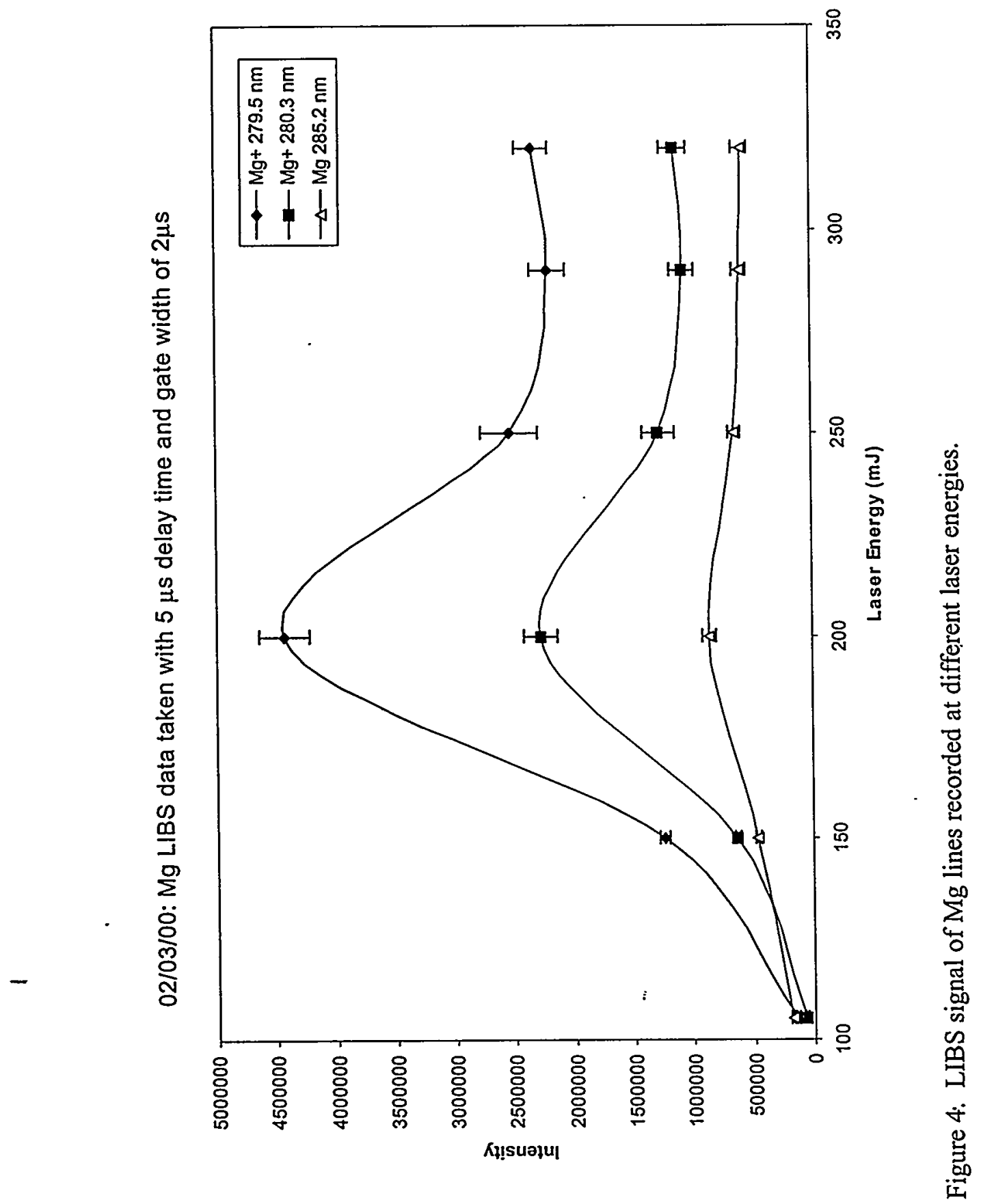




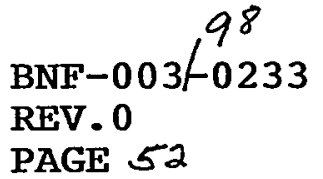

\section{Laser energy}

LIBS spectra were recorded at different laser energies. Figure 4 shows LIBS signal of $\mathrm{Mg}$ lines recorded at different laser energies. The LIBS signal is increased with laser energy till the plasma density become too high which causes more laser energy to be absorbed by the plasma. Therefore less laser energy reaches the sample surface. Thus the intensity of continuum emission is increased and the signal intensity is decreased at high laser energy. The optimized laser energy for jet and bulk liquid measurement is between $150-250 \mathrm{~mJ}$.

\section{Lens-to-surface distance (LTSD)}

LTSD is a critical parameter for the LIBS measurement of solid and liquid sample. A change of the LTSD of a few millimeters can affect the absolute analyte intensity. We found by defocusing the laser beam on the bulk liquid surface, the signal become more reproducible. However, the splash of the liquid due to laser shockwave will disturb the liquid surface and cause the poor precision. The LTSD is more critical in the liquid jet measurement due to its smaller surface. Figure 5 shows LIBS signal at different LTSD with a $20-\mathrm{cm}$ focal length lens. When the LTSD away from the focal length just $1-\mathrm{mm}$, the LIBS signal dropped $\sim 25 \%$. To improve LIBS's precision with a liquid jet system, a longer focal length lens is preferred.

\section{Detection limit}

In both experiments, LIBS signals were optimized for different atomic and ionic lines by adjusting gate delay time and gate width of the detector and also laser energy. LIBS signal of the various elements were recorded at different sample concentrations to obtain the calibration curves at optimized experimental condition. Figure 6 shows LIBS calibrations for $\mathrm{Mn}$ obtained from liquid jet measurement with delay time of $5 \mu$ s and gate of $10 \mu$ s at the laser energy of $200 \mathrm{~mJ}$. The detection limit for $\mathrm{Cr}, \mathrm{Mg}, \mathrm{Mn}$ and $\mathrm{Re}$ were calculated based on the calibration data using

$$
\mathrm{C}_{\mathrm{L}}=30 \text { ó } \mathrm{S}
$$

where ó is standard deviation of the background signal and $S$ is the slope of the calibration curve.

The LOD for $\mathrm{Mn}, \mathrm{Mg}, \mathrm{Cr}$ and $\mathrm{Re}$ in jet measurement were found to be better or comparable than that of the bulk.liquid measurement. Table 2 list the LODs obtained from a liquid jet. The jet system is also preferable than bulk measurement because of the flexibility in changing the solution and easier to obtain optimized LIBS signal without the complication of liquid splashing and maintaining the surface to lens distance.

Table 2. Limit of Detection obtained from a Liquid Jet system

\begin{tabular}{|l|l|l|}
\hline Element' & Wavelength $(\mathrm{nm})$ & LOD $(\mu \mathrm{g} / \mathrm{L})$ \\
\hline $\mathrm{Cr}$ & 425.4 & 400 \\
\hline $\mathrm{Mg}$ & 279.55 & 100 \\
\hline $\mathrm{Mn}$ & 403.076 & 700 \\
\hline $\mathrm{Re}$ & 346.046 & 10000 \\
\hline
\end{tabular}


02/01/00: 10 ppm Cr liquid jet data: Laser:150 mJ; Delay:10 $\mu$ s; Gate:15 $\mu \mathrm{s}$

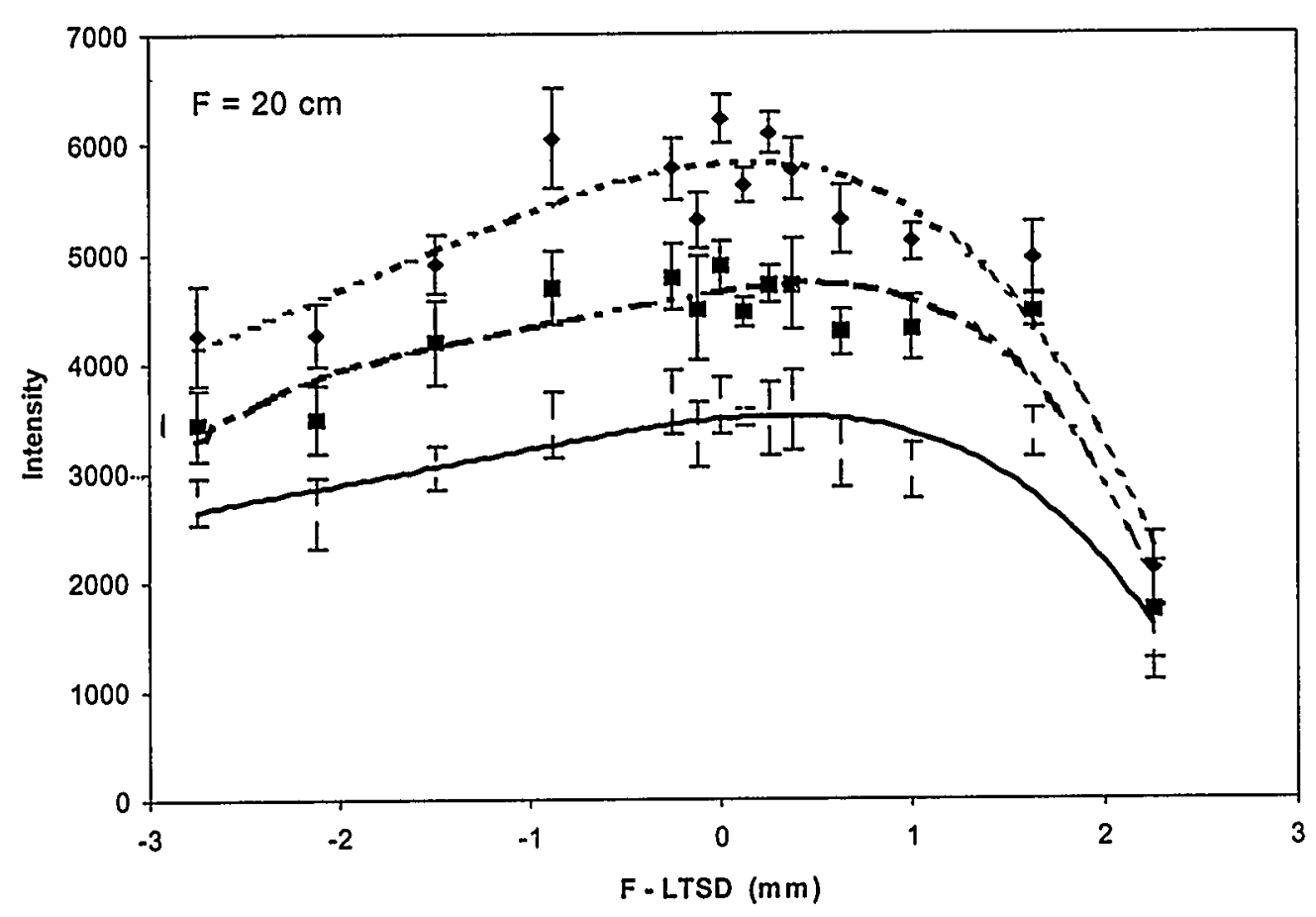

Figure 5. LIBS signal at different LTSD with a $30-\mathrm{cm}$ focal length lens. 
02/14/00: Mn LIBS data with laser energy of $200 \mathrm{~mJ}$ and $5 \mu$ s delay and $10 \mu \mathrm{s}$ gate
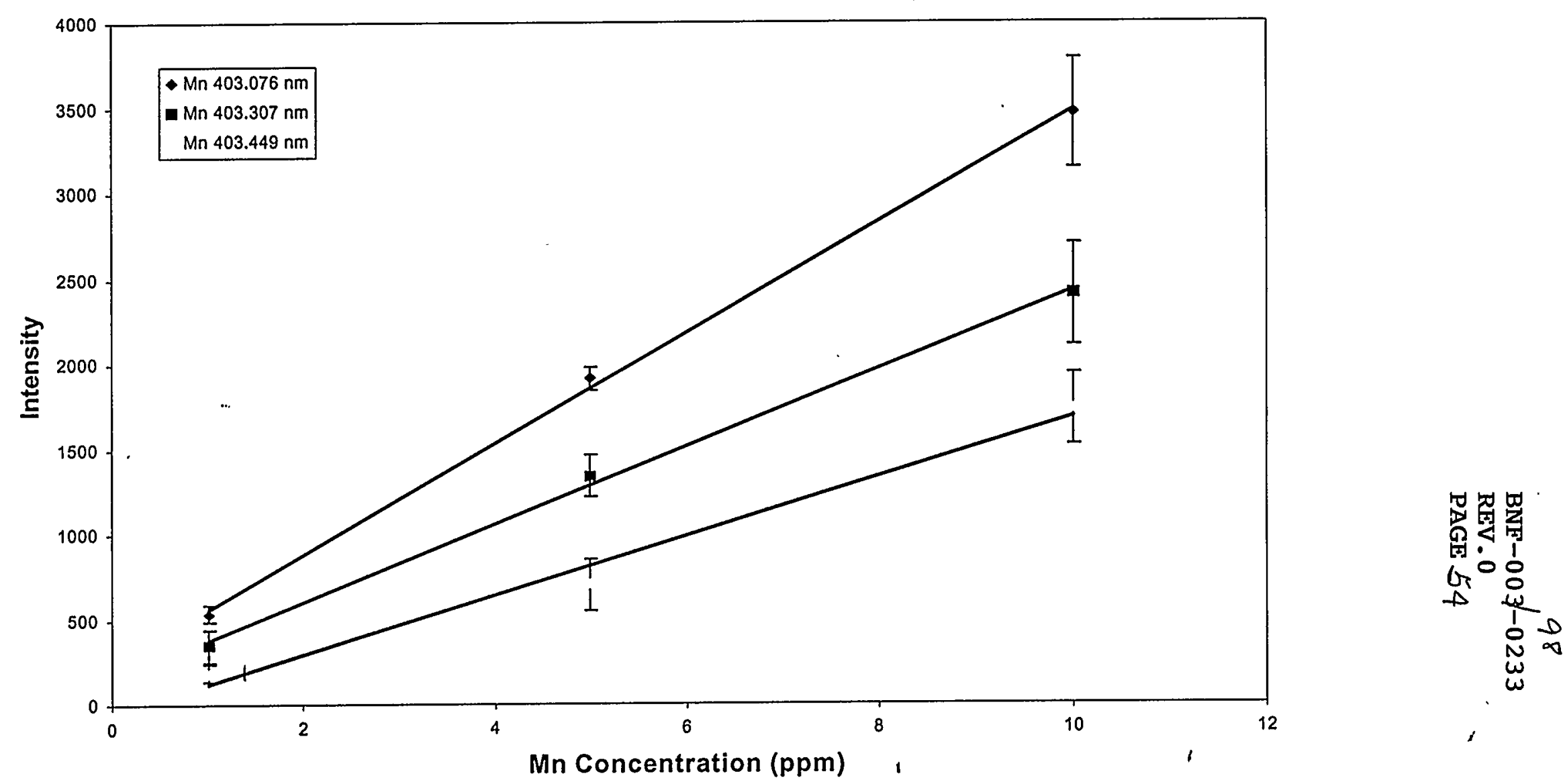

Figure 6. LIBS calibrations for Mn obtained from liquid jet measurement with delay time of $5 \mu$ s and gate of $10 \mu$ s at the laser energy of $200 \mathrm{~mJ}$. 


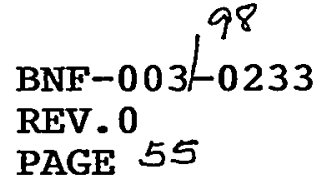

\section{CONCLUSIONS}

A technique is needed to measure Tc during the waste process at DOE Hanford site. LIBS has the potential to be an on-line monitor to monitor Tc in the effluent from the Tc removal column to track the technetium removal process. In this work, we evaluate the analytical figure of merit of LIBS system for the element that has similar properties to Tc. Two experimental setups, bulk liquid and liquid jet, were tested to find the best sampling method for liquid application. We found that liquid jet method is easier to optimize and perform calibration than the bulk liquid method. It is perfect for on line measurement since the fresh sample liquid can continuously supplied. It also will not have the interference from bubbles formed by the preceding spark as in bulk liquid method. The experimental parameters which can affect LIBS' LOD were studied. The calibration data of the test elements were taken at the best experimental parameter for that element. We found that the LOD for all the test elements are better or comparable than that of the bulk liquid measurement.

The emission lines of technetium had reported by Meggars. ${ }^{9}$ The most sensitive lines of Tc were found to be 254.324, 261.0, and $264.702 \mathrm{~nm}$. Current DIAL detection system has reasonable sensitivity in these spectral regions. According to Savannah River Technology Center, the LOD of Tc should be one-order better than LOD of Re. Therefore the estimated LOD for Tc in water is $\sim 1 \mathrm{mg} / \mathrm{L}$. This LOD is still not low enough for Tc monitor. More experiments for improving the sensitivity of the system are needed. A double excitation method that uses two consecutive laser pulses to excite the sample has been shown one to two order signal enhancement in solid and liquid measurement. It might be able to improve LIBS' LOD to achieve the requirement for Tc monitor.

\section{REFERENCES}

1. F.Y. Yueh, J.P. Singh, and H. Zhang, "Elemental Analysis with Laser-induced Breakdown Spectroscopy", Encyclopedia of Analytical Chemistry, John Wiley and Sons, Ltd. Oct. 2000.

2. Radziemski, L.J. and Cremers, D.A. "Spectrochemical Analysis Using Plasma Excitation" in Laser Induced Plasmas and Applications, L.J. Radziemski, and D.A. Cremers, eds. (Marcel Dekker, New York, NY, 1989), Chap. 7, p. 295-326.

3. Singh, J.P., Yueh, F.Y., Zhang H.,1997. Process control and quality 10:247.

4. $\quad$ Singh, J.P., Zhang H., Yueh, F.Y. and Carney, K.P. 1996. Appl. Spectrosc. 50:764.

5. Singh, J.P.; Zhang, H. and Yueh, F.Y. February 1996. Transportable Vitrification System Shakedown Test, Westinghouse Savannah River Corporation and Diagnostic Instrumentation and Analysis Laboratory: Laser-induced Breakdown Spectroscopy Measurements. US DOE Contract No. DE-FG02-93CH-10575. DIAL 10575 Trip Report 96-1.3.

6. Singh, J.P.; Zhang, H. and Yueh, F.Y. April 1996. DOE and EPA Continuous Emission Monitoring Test at EPA National Risk Management Research Laboratory (NRMRL). US DOE Contract No. DE-FG02-93CH-10575. DIAL 10575 Trip Report 96-2.

7. Singh, J.P., Zhang, H., Yueh, F.Y. 1996. "Plasma Arc Centrifugal Treatment PACT-6 Slip Stream Test Bed (SSTB) 100-hour Duration Controlled Emission Demonstration (CED) Test", DIAL Trip Report 96-3.

8. Singh, J.P., Zhang, H., Yueh, F.Y. September 1997. DOE and EPA Continuous Emission Monitoring Test at EPA National Risk Management Research Laboratory (NRMRL), DIAL Trip Report 97-1. 


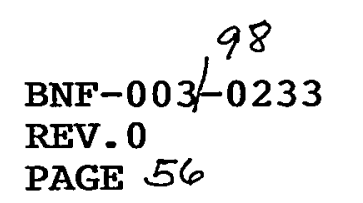

9. Meggars, "The Spectroscopy of Technetium and Promethium", Spectrochemica Acta, $1951,1217-1222$. 